\title{
A single-step genomic model with direct estimation of marker effects
}

\author{
Z. Liu, ${ }^{* 1}$ M. E. Goddard,† F. Reinhardt, ${ }^{*}$ and R. Reents* \\ *Vereinigte Informationssysteme Tierhaltung w.V. (VIT), Heideweg 1, D-27283 Verden, Germany \\ †Melbourne School of Land and Environment, University of Melbourne, Parkville, Victoria 3010, Australia
}

\section{ABSTRACT}

Compared with the currently widely used multi-step genomic models for genomic evaluation, single-step genomic models can provide more accurate genomic evaluation by jointly analyzing phenotypes and genotypes of all animals and can properly correct for the effect of genomic preselection on genetic evaluations. The objectives of this study were to introduce a single-step genomic model, allowing a direct estimation of single nucleotide polymorphism (SNP) effects, and to develop efficient computing algorithms for solving equations of the single-step SNP model. We proposed an alternative to the current single-step genomic model based on the genomic relationship matrix by including an additional step for estimating the effects of SNP markers. Our single-step SNP model allowed flexible modeling of SNP effects in terms of the number and variance of SNP markers. Moreover, our single-step SNP model included a residual polygenic effect with trait-specific variance for reducing inflation in genomic prediction. A kernel calculation of the SNP model involved repeated multiplications of the inverse of the pedigree relationship matrix of genotyped animals with a vector, for which numerical methods such as preconditioned conjugate gradients can be used. For estimating SNP effects, a special updating algorithm was proposed to separate residual polygenic effects from the SNP effects. We extended our single-step SNP model to general multipletrait cases. By taking advantage of a block-diagonal (co)variance matrix of SNP effects, we showed how to estimate multivariate SNP effects in an efficient way. A general prediction formula was derived for candidates without phenotypes, which can be used for frequent, interim genomic evaluations without running the whole genomic evaluation process. We discussed various issues related to implementation of the single-step SNP model in Holstein populations with an across-country genomic reference population.

Received January 9, 2014

Accepted June 10, 2014

${ }^{1}$ Corresponding author: Zengting.Liu@vit.de
Key words: single-step SNP model, genomic prediction, mixed model equation, dairy cattle

\section{INTRODUCTION}

Genomic evaluation and selection based on the theory of Meuwissen et al. (2001) are conducted on a routine basis in an increasing number of countries (VanRaden et al., 2009; Lund et al., 2011). Until now, most countries have applied a so-called multiple-step model to genomic evaluation (VanRaden et al., 2009; Liu et al., 2011), in which a genomic model was fitted to deregressed EBV obtained from a conventional evaluation in a previous step. For German Holsteins, bull EBV from national and multiple-across country evaluation (MACE; Schaeffer, 1994) were deregressed (Jairath et al., 1998) to generate a form of pseudo-phenotype for genomic evaluation in a posterior step. The deregressed EBV were used as phenotypes for German Holsteins in SNP effect estimation (Liu et al., 2011) and also in the step for calculating direct genomic values (DGV) with the EuroGenomics bull reference population (Lund et al., 2011). Pedigree indices for young genotyped animals were calculated using all phenotypes from the whole population. Finally, a selection index method (Mrode, 2005) was used to combine DGV and pedigree index for obtaining genomic estimated breeding values (GEBV).

The multi-step genomic model was relatively simple and easy to implement. A major advantage favoring its application was that the genomic reference population can be optimally set up as desired. For instance, only progeny-tested AI bulls with reliable conventional evaluations were allowed to be included in the reference population, whereas dams of bulls with potentially biased EBV may be excluded. However, the separate steps of estimating SNP effects and conventional evaluation cannot properly account for genomic preselection and thus conventional EBV, and subsequently GEBV, would be biased (Patry and Ducrocq, 2011). The pseudo-phenotype, deregressed EBV (Liu, 2011), may have extreme values as a result of low information content; for example, some bulls with limited daughter information may have very low or high deregressed EBV.

In contrast to the multi-step models, single-step genomic models (Aguilar et al., 2010; Christensen and 
Lund, 2010; Gao et al., 2012; Koivula et al., 2012; Su et al., 2012; Ødegård et al., 2013) evaluate genotyped and nongenotyped animals jointly, and thus can give unbiased predictions of genetic merits. The single-step models can perfectly account for the effect of genomic preselection because culled candidates can be included in the evaluation. However, single-step genomic models do not directly provide estimates of SNP effects, which give meaningful biological interpretation of SNP genotypes on phenotypes and for calculating DGV. Additionally, breeding organizations or individual breeders may want to know genomic evaluation results as soon as their candidate animals have been genotyped, in order to make immediate selection decisions and reduce the cost of genomic selection. The current single-step genomic model based on the genomic relationship matrix (Aguilar et al., 2010; Christensen and Lund, 2010) typically evaluates all animals together, even when no new phenotypes have been added. Therefore, a simple and fast computing algorithm needs to be developed for timely interim genomic evaluation of newly genotyped candidates.

In conventional genetic evaluation, at least for German Holsteins, a group of correlated traits have been evaluated jointly using a multiple trait model; for example, heifer and cow female fertility traits or all linear traits of conformation. However, a single-trait genomic model has typically been applied to deregressed EBV in a multi-step genomic evaluation (Liu et al., 2011). To properly consider correlated phenotypic information between the traits, single-step genomic models must be extended to evaluate multiple traits simultaneously (Aguilar et al., 2011b; Calus and Veerkamp, 2011; Christensen et al., 2012). This extension is required for estimating genomic breeding values as well as SNP effects.

Conventional routine genetic evaluation considers all cows with phenotypes typically born in last $20 \mathrm{yr}$ or older and all animals in recorded pedigree, but only a small fraction of the evaluated animals have been genotyped. Therefore, the number of animals evaluated with a multi-step genomic model was much smaller than that in a conventional genetic evaluation. This means that the upgrading of the current multi-step genomic model to a single-step genomic model must deal with a much larger number of animals. Efficient computing algorithms are thus needed to jointly estimate additive genetic effects of cows with phenotypes, genotyped animals, and their relatives in pedigree.

The objectives of our study were (1) to introduce a single-step genomic model allowing a direct estimation of SNP marker effects, (2) to extend the single-step SNP model to a multiple-trait evaluation, (3) to develop simple formulas for fast interim genomic evaluation of candidates, and (4) to develop efficient computational algorithms for estimating effects of the new model.

\section{MATERIALS AND METHODS}

\section{A Single-Step SNP Model}

It is assumed that there are 2 groups of animals to be evaluated: group 1 (without genotype data) and group 2 (with genotype data available). The genotyped animals may or may not have phenotypic records. A mixed linear model is used for analyzing phenotypic data of both groups:

$$
\mathbf{y}=\mathbf{X b}+\mathbf{Z}_{p} \mathbf{p}+\mathbf{W u}+\mathbf{e},
$$

where $\mathbf{y}$ is an $n_{T} \times 1$ vector of phenotypic records, $n_{T}$ $=$ number of phenotype records; $\mathbf{b}$ is an $n_{F} \times 1$ vector of all fixed effects, $n_{F}=$ number of all fixed effects; $\mathbf{p}$ is an $n_{p} \times 1$ vector of nongenetic random effects (e.g., permanent environmental effects of cows), $n_{p}=$ number of cows with phenotype data $p ; \mathbf{u}$ is an $n \times 1$ vector of additive genetic effects, $n=$ number of animals; $\mathbf{e}$ is an $n_{T} \times 1$ vector of residual effects; and $\mathbf{X}$ of order $n_{T}$ $\times n_{F}, \mathbf{Z}_{p}$ of order $n_{T} \times n_{p}$, and $\mathbf{W}$ of order $n_{T} \times n$ are incidence matrices for effects $\mathbf{b}, \mathbf{p}$, and $\mathbf{u}$, respectively.

Without loss of generality, we assume throughout this paper that

$$
\operatorname{var}(\mathbf{p})=\mathbf{I} \sigma_{p}^{2} \text { and } \operatorname{var}(\mathbf{e})=\mathbf{I} \sigma_{e}^{2}
$$

(where $\sigma_{p}^{2}$ is variance of permanent environmental effects and $\sigma_{e}^{2}$ is residual variance), although more complex (co)variance structures for $\mathbf{p}$ and e could also be considered. We further assume that $m$ SNP markers selected from a SNP chip for genomic evaluation cannot explain all additive genetic variance $\left(\sigma_{u}^{2}\right)$, leaving $k$ $\sigma_{u}^{2}$ residual polygenic variance, where $k$ is the proportion of additive genetic variance not explained by all the $m$ SNP markers. Thus, additive genetic effects of the genotyped animals in group 2 can be divided into:

$$
\mathbf{u}_{2}=\mathbf{Z g}+\mathbf{a}_{2},
$$

where $\mathbf{u}_{2}=n_{g} \times 1$ vector of additive genetic effects of the genotyped animals, $n_{g}=$ number of genotyped animals; $\mathbf{g}=m \times 1$ vector of additive genetic effects of the $m$ fitted SNP markers; $\mathbf{a}_{2}=n_{g} \times 1$ vector of residual polygenic effects (RPG) of the genotyped animals; and $\mathbf{Z}=$ a design matrix of order $n_{g} \times m$, containing genotype indicators $(-1$ and 1 for 2 homozygote and 0 for heterozygote genotypes) of the genotyped animals at all $m$ SNP markers. 
Residual polygenic variance parameter $k$ may take any value between 0 and 1 but excluding 2 boundary values $k=0$ and $k=1$.

It is assumed that the SNP marker effects have a (co) variance structure

$$
\operatorname{var}(\mathbf{g})=\mathbf{B} \sigma_{u}^{2}
$$

Under the assumption of uncorrelated SNP effects, B is a diagonal matrix of order of $m \times m$. If all the $m$ SNP markers explain equal additive genetic variance such as in a BLUP SNP model (Meuwissen et al., 2001; Liu et al., 2011), then

$$
\mathbf{B}=\frac{1-k}{m} \mathbf{I}
$$

Alternatively, a Bayesian genomic model such as Bayes A or B (Meuwissen et al., 2001), Bayes C (Habier et al., 2011), or Bayes R (Erbe et al., 2012) can be fitted to the SNP effects, allowing some SNP markers have more variance than others. Theoretically, the SNP effects could also be assumed to be correlated using a full matrix B (Szyda et al., 2009). Additionally, the number of fitted SNP markers, $m$, depends on the assumptions of the SNP models. However, the sum of the SNP effects, also known as DGV, must follow a normal distribution.

Relationships between $n-n_{g}$ nongenotyped animals in group 1 and $n_{g}$ genotyped animals in group 2 are defined as

$$
\mathbf{A}=\left[\begin{array}{ll}
\mathbf{A}_{11} & \mathbf{A}_{12} \\
\mathbf{A}_{21} & \mathbf{A}_{22}
\end{array}\right]
$$

where the submatrix $\mathbf{A}_{11}$ or $\mathbf{A}_{22}$ represents pedigree relationship between nongenotyped animals or between genotyped animals, respectively, and the submatrix $\mathbf{A}_{12}$ represents pedigree relationship between genotyped and nongenotyped animals.For RPG of the genotyped animals,

$$
\operatorname{var}\left(\mathbf{a}_{2}\right)=\mathbf{A}_{22} k \sigma_{u}^{2},
$$

and under an assumption of zero covariance

$$
\operatorname{Cov}\left(\mathbf{g}, \mathbf{a}_{2}\right)=\mathbf{0},
$$

we have

$$
\operatorname{var}\left(\mathbf{u}_{2}\right)=\operatorname{var}\left(\mathbf{Z g}+\mathbf{a}_{2}\right)=\left(\mathbf{Z B Z}^{\prime}+k \mathbf{A}_{22}\right) \sigma_{u}^{2}=\mathbf{G}_{22} \sigma_{u}^{2} .
$$

From Equation [9], we can see that our genomic relationship matrix $\mathbf{G}_{22}$ is a linear function of observed genomic relationship matrix $\mathbf{Z B Z}^{\prime}$ with an implicit weight $(1-k)$ and the expected pedigree relationship matrix $\mathbf{A}_{22}$ with a weight $k$. (Co)variance matrix of additive genetic effects of nongenotyped animals in group $1\left(\mathbf{u}_{1}\right)$ and genotyped animals $\left(\mathbf{u}_{2}\right)$ is

$$
\operatorname{var}(\mathbf{u})=\operatorname{var}\left(\begin{array}{l}
\mathbf{u}_{1} \\
\mathbf{u}_{2}
\end{array}\right)=\mathbf{G} \sigma_{u}^{2}
$$

and inverse of matrix $\mathbf{G}$ (see Appendix A for derivation) is

$\mathbf{G}^{-1}=\left[\begin{array}{cc}\mathbf{A}^{11} & \mathbf{A}^{12} \\ \mathbf{A}^{21} & \mathbf{G}_{22}^{-1}+\mathbf{A}^{22}-\mathbf{A}_{22}^{-1}\end{array}\right]=\left[\mathbf{A}^{-1}+\left[\begin{array}{cc}0 & 0 \\ 0 & \mathbf{G}_{22}^{-1}-\mathbf{A}_{22}^{-1}\end{array}\right]\right.$.

Appending the vector of SNP effects $\mathbf{g}$ to $\mathbf{u}$ gives a vector $\mathbf{h}$, which has a (co)variance matrix of order $(n$ $+m) \times(n+m)$ :

$$
\operatorname{var}(\mathbf{h})=\operatorname{var}\left[\begin{array}{c}
\mathbf{u}_{1} \\
\mathbf{u}_{2} \\
\mathbf{g}
\end{array}\right]=\mathbf{H} \sigma_{u}^{2}
$$

and inverse of matrix $\mathbf{H}$ (see Appendix A for derivation) is

$$
\mathbf{H}^{-1}=\left[\begin{array}{ccc}
\mathbf{A}^{11} & \mathbf{A}^{12} & \mathbf{0} \\
\mathbf{A}^{21} & \mathbf{A}^{22}+\left(\frac{1}{k}-1\right) \mathbf{A}_{22}^{-1} & -\frac{1}{k} \mathbf{A}_{22}^{-1} \mathbf{Z} \\
\mathbf{0} & -\frac{1}{k} \mathbf{Z}^{\prime} \mathbf{A}_{22}^{-1} & \mathbf{B}^{-1}+\frac{1}{k} \mathbf{Z}^{\prime} \mathbf{A}_{22}^{-1} \mathbf{Z}
\end{array}\right] .
$$

Note that the inverse matrix $\mathbf{H}^{-1}$ requires $k>0$. Replacing the inverse of pedigree relationship matrix in Henderson's mixed model equations (MME) with $\mathbf{H}^{-1}$ gives MME for all the effects of model [Eq. 1]:

$$
\left[\begin{array}{ccc}
\mathbf{X}^{\prime} \mathbf{X} & \mathbf{X}^{\prime} \mathbf{Z}_{p} & \mathbf{X}^{\prime} \mathbf{W} \\
\mathbf{Z}_{p}{ }^{\prime} \mathbf{X} & \mathbf{Z}_{p}{ }^{\prime} \mathbf{Z}_{p}+\mathbf{I} \delta & \mathbf{Z}_{p}{ }^{\prime} \mathbf{W} \\
\mathbf{W}^{\prime} \mathbf{X} & \mathbf{W}^{\prime} \mathbf{Z}_{p} & \mathbf{W}^{\prime} \mathbf{W}+\mathbf{H}^{-1} \lambda
\end{array}\right]\left[\begin{array}{c}
\hat{\mathbf{b}} \\
\hat{\mathbf{p}} \\
\hat{\mathbf{h}}
\end{array}\right]=\left[\begin{array}{c}
\mathbf{X}^{\prime} \mathbf{y} \\
\mathbf{Z}_{p}{ }^{\prime} \mathbf{y} \\
\mathbf{W}^{\prime} \mathbf{y}
\end{array}\right],[14
$$

where $\delta=\sigma_{e}^{2} / \sigma_{p}^{2}$ and $\lambda=\sigma_{e}^{2} / \sigma_{u}^{2}$.

Replacing $\mathbf{H}^{-1}$ in above Equation [14] with Equation [13] leads to: 


$$
\left[\begin{array}{ccccc}
\mathbf{X}^{\prime} \mathbf{X} & \mathbf{X}^{\prime} \mathbf{Z}_{p} & \mathbf{X}^{\prime} \mathbf{W}_{1} & \mathbf{X}^{\prime} \mathbf{W}_{2} & \mathbf{0} \\
\mathbf{Z}_{p}{ }^{\prime} \mathbf{X} & \mathbf{Z}_{p}{ }^{\prime} \mathbf{Z}_{p}+\mathbf{I} \delta & \mathbf{Z}_{p}{ }^{\prime} \mathbf{W}_{1} & \mathbf{Z}_{p}{ }^{\prime} \mathbf{W}_{2} & \mathbf{0} \\
\mathbf{W}_{1}{ }^{\prime} \mathbf{X} & \mathbf{W}_{1} \mathbf{Z}_{p} & \mathbf{W}_{1}{ }^{\prime} \mathbf{W}_{1}+\lambda \mathbf{A}^{11} & \lambda \mathbf{A}^{12} & \mathbf{0} \\
\mathbf{W}_{2}{ }^{\prime} \mathbf{X} & \mathbf{W}_{2}{ }^{\prime} \mathbf{Z}_{p} & \lambda \mathbf{A}^{21} & \mathbf{W}_{2}{ }^{\prime} \mathbf{W}_{2}+\lambda\left(\mathbf{A}^{22}+\left(\frac{1}{k}-1\right) \mathbf{A}_{22}^{-1}\right) & -\frac{1}{k} \lambda \mathbf{A}_{22}^{-1} \mathbf{Z} \\
\mathbf{0} & \mathbf{0} & \mathbf{0} & -\frac{1}{k} \lambda \mathbf{Z}^{\prime} \mathbf{A}_{22}^{-1} & \lambda\left(\mathbf{B}^{-1}+\frac{1}{k} \mathbf{Z}^{\prime} \mathbf{A}_{22}^{-1} \mathbf{Z}\right)
\end{array}\right]\left[\begin{array}{c}
\hat{\mathbf{b}} \\
\hat{\mathbf{p}} \\
\hat{\mathbf{u}}_{1} \\
\hat{\mathbf{u}}_{2} \\
\hat{\mathbf{g}}
\end{array}\right]=\left[\begin{array}{c}
\mathbf{X}^{\prime} \mathbf{y} \\
\mathbf{Z}_{p}{ }^{\prime} \mathbf{y} \\
\mathbf{W}_{1}{ }^{\prime} \mathbf{y} \\
\mathbf{W}_{2}{ }^{\prime} \mathbf{y} \\
\mathbf{0}
\end{array}\right],
$$

where $\mathbf{W}_{1}$ of order $n_{T} \times\left(n-n_{g}\right)$ and $\mathbf{W}_{2}$ of order $n_{T} \times n_{g}$ are incidence matrices for additive genetic effects of the nongenotyped and genotyped animals, respectively. Above, Equation [15] can be rearranged into 2 sets that are solved iteratively:

$$
\begin{gathered}
{\left[\begin{array}{cccc}
\mathbf{X}^{\prime} \mathbf{X} & \mathbf{X}^{\prime} \mathbf{Z}_{p} & \mathbf{X}^{\prime} \mathbf{W}_{1} & \mathbf{X}^{\prime} \mathbf{W}_{2} \\
\mathbf{Z}_{p}{ }^{\prime} \mathbf{X} & \mathbf{Z}_{p}{ }^{\prime} \mathbf{Z}_{p}+\mathbf{I} \delta & \mathbf{Z}_{p}{ }^{\prime} \mathbf{W}_{1} & \mathbf{Z}_{p}{ }^{\prime} \mathbf{W}_{2} \\
\mathbf{W}_{1}{ }^{\prime} \mathbf{X} & \mathbf{W}_{1}^{\prime} \mathbf{Z}_{p} & \mathbf{W}_{1}{ }^{\prime} \mathbf{W}_{1}+\lambda \mathbf{A}^{11} & \lambda \mathbf{A}^{12} \\
\mathbf{W}_{2}{ }^{\prime} \mathbf{X} & \mathbf{W}_{2}{ }^{\prime} \mathbf{Z}_{p} & \lambda \mathbf{A}^{21} & \mathbf{W}_{2}{ }^{\prime} \mathbf{W}_{2}+\lambda\left(\mathbf{A}^{22}+\left(\frac{1}{k}-1\right) \mathbf{A}_{22}^{-1}\right)
\end{array}\right]\left[\begin{array}{c}
\hat{\mathbf{b}} \\
\hat{\mathbf{p}} \\
\hat{\mathbf{u}}_{1} \\
\hat{\mathbf{u}}_{2}
\end{array}\right]=\left[\begin{array}{c}
\mathbf{X}^{\prime} \mathbf{y} \\
\mathbf{Z}_{p}{ }^{\prime} \mathbf{y} \\
\mathbf{W}_{1}{ }^{\prime} \mathbf{y} \\
\mathbf{W}_{2}{ }^{\prime} \mathbf{y}+\frac{1}{k} \lambda \mathbf{A}_{22}^{-1} \mathbf{Z} \hat{\mathbf{g}}
\end{array}\right],} \\
\left(\mathbf{B}^{-1}+\frac{1}{k} \mathbf{Z}^{\prime} \mathbf{A}_{22}^{-1} \mathbf{Z}\right) \hat{\mathbf{g}}=\frac{1}{k} \mathbf{Z}^{\prime} \mathbf{A}_{22}^{-1} \hat{\mathbf{u}}_{2} .
\end{gathered}
$$

\section{Computing Strategies for Solving the Equations}

In the literature, several computing strategies have been developed for solving MME of single-step genomic models (Misztal et al., 2009; Legarra and Ducrocq, 2012). Here we focus on solving MME [Eq. 16] and [Eq. 17] of our single-step SNP model [Eq. 1].

Rearrange the $\mathbf{u}_{2}$ equation in [Eq. 16] so that only the conventional MME terms are kept on the left-hand side and all genomic terms are moved to the right-hand side (RHS):

$$
\begin{aligned}
& \mathbf{W}_{2}{ }^{\prime} \mathbf{X} \hat{\mathbf{b}}+\mathbf{W}_{2}{ }^{\prime} \mathbf{Z}_{p} \hat{\mathbf{p}}+\lambda \mathbf{A}^{21} \hat{\mathbf{u}}_{1}+\left(\mathbf{W}_{2}{ }^{\prime} \mathbf{W}_{2}+\lambda \mathbf{A}^{22}\right) \hat{\mathbf{u}}_{2} \\
& =\mathbf{W}_{2}{ }^{\prime} \mathbf{y}+\frac{1}{k} \lambda \mathbf{A}_{22}^{-1} \mathbf{Z} \hat{\mathbf{g}}+\lambda\left(1-\frac{1}{k}\right) \mathbf{A}_{22}^{-1} \hat{\mathbf{u}}_{2} .
\end{aligned}
$$

Because

$$
\begin{gathered}
\mathbf{a}_{2}=\mathbf{u}_{2}-\mathbf{Z g}, \\
\mathbf{W}_{2}{ }^{\prime} \mathbf{X} \hat{\mathbf{b}}+\mathbf{W}_{2}{ }^{\prime} \mathbf{Z}_{p} \hat{\mathbf{p}}+\lambda \mathbf{A}^{21} \hat{\mathbf{u}}_{1}+\left(\mathbf{W}_{2}{ }^{\prime} \mathbf{W}_{2}+\lambda \mathbf{A}^{22}\right) \hat{\mathbf{u}}_{2}[18] \\
=\mathbf{W}_{2}{ }^{\prime} \mathbf{y}+\lambda \mathbf{A}_{22}^{-1}\left(\hat{\mathbf{u}}_{2}-\frac{1}{k} \hat{\mathbf{a}}_{2}\right) .
\end{gathered}
$$

Because it is infeasible to obtain the inverse matrix $\mathbf{A}_{22}^{-1}$ for a large population such as German Holsteins, with hundreds of thousands of genotyped animals, indirect ways such as iteration on data technique (Schaeffer and Kennedy, 1986) have to be applied for routine genomic evaluation. Following the idea of Legarra and Ducrocq (2012), the genomic contribution term

$$
\mathbf{A}_{22}^{-1}\left(\hat{\mathbf{u}}_{2}-\frac{1}{k} \hat{\mathbf{a}}_{2}\right)=\mathbf{A}_{22}^{-1} \hat{\mathbf{u}}_{2}^{*}=\hat{\phi}
$$

can be computed equivalently by solving the following equation:

$$
\mathbf{A}_{22} \hat{\phi}=\hat{\mathbf{u}}_{2}^{*} .
$$

From Equation [18], we can see that RHS of conventional MME needs to be corrected for the genomic contribution $\lambda \hat{\phi}$.

Rearranging the terms of SNP effects in Equation [17] gives

$$
\hat{\mathbf{g}}=\frac{1}{k} \mathbf{B Z}^{\prime} \mathbf{A}_{22}^{-1}\left(\hat{\mathbf{u}}_{2}-\mathbf{Z} \hat{\mathbf{g}}\right)=\frac{1}{k} \mathbf{B} \mathbf{Z}^{\prime} \mathbf{A}_{22}^{-1} \hat{\mathbf{a}}_{2}=\frac{1}{k} \mathbf{B} \mathbf{Z}^{\prime} \hat{\gamma},[21]
$$

where

$$
\hat{\gamma}=\mathbf{A}_{22}^{-1} \hat{\mathbf{a}}_{2} .
$$

Analogous to solving for $\hat{\phi}, \hat{\gamma}$ can be obtained by solving

$$
\mathbf{A}_{22} \hat{\gamma}=\hat{\mathbf{a}}_{2}
$$


Equation [19] for $\hat{\phi}$ and Equation [22] for $\hat{\gamma}$ represent 2 extra major calculations for genomic contribution of the single-step SNP model [Eq. 1]. Numerical iterative procedures, such as Gauss-Jacobi algorithm (P. VanRaden, USDA Animal Improvement Programs Laboratory, Beltsville, MD; personal communication) or preconditioned conjugate gradients (Strandén and Lidauer, 1999), may be used for solving Equations [20] and [23] to get $\hat{\phi}$ and $\hat{\gamma}$. The computing algorithm by Aguilar et al. (2011a) can be used here to calculate $\mathbf{A}_{22} \hat{\phi}$ or $\mathbf{A}_{22} \hat{\gamma}$ without storing or setting up matrix $\mathbf{A}_{22}$ explicitly.

For solving the conventional parts of MME in Equation [16], Gauss-Seidel algorithm or preconditioned conjugate gradient algorithm (Strandén and Lidauer, 1999) may be used together with iteration on data technique (Schaeffer and Kennedy, 1986). For genotyped animals, the genomic contribution [Eq. 19] to the RHS of Equation [18] is calculated by solving Equation [20] in a second step. Additionally, SNP effects are estimated with Equation [21] (see below for details), with $\hat{\gamma}$ obtained by iteratively solving Equation [23]. These 3 steps are iterated until all effects in Equations [16] and [17] are converged. The convergence criterion was defined as the sum of squared differences in the effect estimates between 2 consecutive rounds of iteration divided by the sum of squares of the estimates from current round of iteration.

\section{Estimating SNP Effects with a Special Algorithm}

In contrast to single-step genomic BLUP (SSGblup) models (Aguilar et al., 2010; Legarra and Ducrocq, 2012), our genomic model [Eq. 1] has an extra step of estimating SNP effects. This extra step enables us to actively control the information flow from genomic reference population to candidates. Genomic prediction can be improved, based on our experience with a multiple-step genomic model (Liu et al., 2011), if bulls are removed from the reference population that have less reliable, potentially biased, conventional EBV due to limited data from daughters. Second, (selectively) genotyped bull dams or elite cows with possibly preferential treatments should be kept from the reference population as well. Third, genotyped animals with imputed genotypes from low-density chips may be treated as less accurate than reference animals genotyped with a standard chip. Last, one may wish to exclude genotyped non-Holstein animals in a Holstein reference population in a joint multi-breed genetic evaluation. For instance, a much smaller number of cows of the Jersey breed are jointly evaluated with predominantly Holstein cows for milk production traits in Germany (Liu et al., 2004), but we prefer excluding those genotyped Jersey bulls from the
German Holstein genomic reference population for genomic prediction of Holstein animals. To decide which genotyped animals are allowed to contribute to SNP effect estimation, we introduce a filter matrix $\mathbf{F}$ to all $n_{g}$ genotyped animals:

$$
\mathbf{F}=\operatorname{diag}\{1,0,0,1, \cdots, 1,1,0\}
$$

where diagonal element 1 or 0 means a genotyped animal defined as a reference animal or not, respectively. The $\mathbf{F}$ matrix can be inserted into the SNP effect estimation Equation [21]:

$$
\hat{\mathbf{g}}=\frac{1}{k} \mathbf{B Z}^{\prime} \mathbf{F} \mathbf{A}_{22}^{-1} \hat{\mathbf{a}}_{2},
$$

to allow additive genetic effects of only selected genotyped animals to be used in the conversion to SNP effect estimates. Alternatively, very small values for the F matrix may be assigned to animals with problematic phenotypes. Because all genotyped animals are included in $\mathbf{A}_{22}^{-1} \hat{\mathbf{a}}_{2}$, the potential bias caused by genomic preselection found in a multistep genomic model (Patry and Ducrocq, 2011) should not exist in either the additive genetic effects $\hat{\mathbf{u}}_{2}$ and $\hat{\mathbf{a}}_{2}$, or in the SNP effect estimates $\hat{\mathbf{g}}$, despite the selection of some reference from genotyped animals.

Estimating SNP effects represents a so-called large $p$ and small $n$ computational issue, where more SNP effects are to be estimated using fewer reference animals. The RPG, $\hat{\mathbf{a}}_{2}$, of reference animals need to be separated from $\hat{\mathbf{u}}_{2}$ to obtain SNP effect estimates, $\hat{\mathbf{g}}$. To separate both components of $\hat{\mathbf{u}}_{2}$, an iterative procedure is needed. For a given set of $\hat{\mathbf{u}}_{2}$ estimates of reference animals, the following relationships exist between 2 consecutive rounds $(r)$ of iteration:

$$
\begin{gathered}
\mathbf{Z}\left(\hat{\mathbf{g}}^{[r+1]}-\hat{\mathbf{g}}^{[r]}\right)=\left(\hat{\mathbf{u}}_{2}-\hat{\mathbf{a}}_{2}^{[r+1]}\right)-\left(\hat{\mathbf{u}}_{2}-\hat{\mathbf{a}}_{2}^{[r]}\right)=\hat{\mathbf{a}}_{2}^{[r]}-\hat{\mathbf{a}}_{2}^{[r+1]}, \\
\hat{\mathbf{a}}_{2}^{[r+1]}=\hat{\mathbf{a}}_{2}^{[r]}-\mathbf{Z}\left(\hat{\mathbf{g}}^{[r+1]}-\hat{\mathbf{g}}^{[r]}\right) .
\end{gathered}
$$

Equation [27] shows that, for a given set of $\hat{\mathbf{u}}_{2}$, the RPG $\hat{\mathbf{a}}_{2}$ can be updated using the changes in SNP effect estimates between the 2 rounds $r+1$ and $r$. Iterating Equation [25] for SNP effects and Equation [27] for RPG, both effects can be estimated efficiently at a given set $\hat{\mathbf{u}}_{2}$ of reference animals, because the updating of RPG involves reading genotypes of reference animals only once. As Equation [25] estimates effects of all SNP markers simultaneously, the order of SNP markers no longer plays a role in convergence behavior, as observed 
by Liu et al. (2011) with a SNP effect estimation algorithm on a SNP-by-SNP basis.

\section{A Multiple-Trait Single-Step SNP Model}

In conventional genetic evaluations of German Holsteins, all trait groups have been evaluated with a multitrait model; for example, a multiple-trait model for 5 female fertility traits, or a multiple-trait model with a correlated maternal effect for 2 calving traits. To keep the optimal properties of the multiple-trait models (Aguilar et al., 2011b; Calus and Veerkamp, 2011; Christensen et al., 2012), the single-step SNP model [Eq. 1] needs to be extended to evaluate multiple traits simultaneously.

It is assumed that a total number of $\mathrm{T}$ traits $(t=$ $1, \ldots, \mathrm{T}$ ) are correlated with a genetic (co)variance matrix:

$$
\begin{aligned}
\mathbf{s R}_{G} \mathbf{s}^{\prime} & =\left[\begin{array}{c}
\sigma_{u_{1}} \\
\sigma_{u_{2}} \\
\vdots \\
\sigma_{u_{T}}
\end{array}\right]\left[\begin{array}{cccc}
1 & r_{12} & \cdots & r_{1 T} \\
& 1 & \cdots & r_{2 T} \\
& & \ddots & \vdots \\
\text { symm. } & & & 1
\end{array}\right]\left[\begin{array}{llll}
\sigma_{u_{1}} & \sigma_{u_{2}} & \cdots & \sigma_{u_{T}}
\end{array}\right] \\
& =\left[\begin{array}{cccc}
\sigma_{u_{1}}^{2} & r_{12} \sigma_{u_{1}} \sigma_{u_{2}} & \cdots & r_{1 T} \sigma_{u_{1}} \sigma_{u_{T}} \\
& \sigma_{u_{2}}^{2} & \cdots & r_{2 T} \sigma_{u_{2}} \sigma_{u_{T}} \\
& & \ddots & \vdots \\
\text { symm. } & & & \sigma_{u_{T}}^{2}
\end{array}\right],
\end{aligned}
$$

where $\mathbf{R}_{G}$ is a genetic correlation matrix, $\mathbf{s}$ is a vector of additive genetic standard deviations of the traits, $\sigma_{u_{t}}$ is additive genetic standard deviation of trait $t$, and $r_{t t^{\prime}}$ is genetic correlation between traits $t$ and $t^{\prime}$.

If SNP effects are reordered by traits nested within SNP marker, then the (co)variance matrix of the SNP effects in Equation [5] has a block-diagonal matrix structure:

$$
\operatorname{var}(\mathbf{g})=\left[\begin{array}{cccc}
\mathbf{s B}_{11} \mathbf{s}^{\prime} & & & \mathbf{0} \\
& \mathbf{s B}_{22} \mathbf{s}^{\prime} & & \\
& & \ddots & \\
\mathbf{0} & & & \mathbf{s B}_{m m} \mathbf{s}^{\prime}
\end{array}\right]
$$

where $\mathbf{B}_{j j}$ for SNP marker $j(j=1, \ldots, m)$ is under the BLUP SNP model:

$$
\mathbf{B}_{j j}=\frac{1-k}{m}\left[\begin{array}{cccc}
1 & r_{12} & \cdots & r_{1 T} \\
& 1 & \cdots & r_{2 T} \\
& & \ddots & \vdots \\
\text { symm. } & & & 1
\end{array}\right] .
$$

The correlations of SNP effects between traits in Equation [30] are assumed to be equal to the genetic correlations between traits on the genome level as in Equation [28]. This assumption can hold only if the same residual polygenic variance parameter $k$ is assumed for all the T traits in the multiple-trait BLUP SNP model [Eq. 5], which assumes equal SNP variances. If the residual polygenic variance parameter $k$ could vary among the $\mathrm{T}$ traits, the correlations of SNP effects in [30] may not be equal to those in Equation [28], and the matrix $\mathbf{B}_{j j}$ may have a much more complex structure. In this case, the off-diagonal element of $\mathbf{s B}_{j j} \mathbf{s}^{\prime}$ for a SNP marker $j(j$ $=1, \ldots, m)$ between traits $t$ and $t^{\prime}$ may be approximated as

$$
\left\{\mathbf{s B}_{j j^{\prime}} \mathbf{s}^{\prime}\right\}_{t, t^{\prime}}=\left\{\frac{1-k_{t}}{m} \frac{1-k_{t^{\prime}}}{m} r_{t, t^{\prime}} \sigma_{u_{t}} \sigma_{u_{t^{\prime}}}\right\}
$$

Based on the experience with German Holstein genomic evaluations (Liu et al., 2011), the assumption of equal $k$ parameter for all subtraits in a multiple-trait model is realistic for most trait groups in routine genomic evaluations. Also, under the SSGblup model (Aguilar et al., 2011b), the same values used for weighting the observed genomic relationship matrix and expected pedigree relationship matrix are applied to all subtraits in a multi-trait genomic evaluation. In contrast to the BLUP SNP model [Eq. 5], Bayesian SNP models allowing some SNP having no effects in some traits may have a much more complex structure of SNP effect correlations among the traits than Equation [30].

Corresponding to the SNP effects ordered by traits within SNP marker, design matrix $\mathbf{Z}$ of the multi-trait model for $n$ genotyped animals genotyped at $m$ SNP markers is

$$
\mathbf{Z}=\left[\begin{array}{llll}
\mathbf{Z}_{1} & \mathbf{Z}_{2} & \cdots & \mathbf{Z}_{m}
\end{array}\right],
$$

where, for the $j$ th SNP, the design matrix $\mathrm{Z}_{j}$ with dimensions $n_{T} \times \mathrm{T}$ is

$$
\mathbf{Z}_{j}{ }^{\prime}=\left[\begin{array}{lllll}
\mathbf{Z}_{1 j}{ }^{\prime} & \vdots & \mathbf{Z}_{i j}{ }^{\prime} & \vdots & \mathbf{Z}_{n j}{ }^{\prime}
\end{array}\right],
$$

with a submatrix of $\mathrm{Z}_{i j}$ for animal $i(i=1, \ldots, n)$ being 


$$
\mathbf{Z}_{i j}=z_{i j} \mathbf{J}=z_{i j}\left[\begin{array}{cccc}
1 & 1 & \cdots & 1 \\
1 & 1 & \cdots & 1 \\
\vdots & \vdots & \ddots & \vdots \\
1 & 1 & \cdots & 1
\end{array}\right]
$$

where $z_{i j}$ is genotype value of SNP $j$ of animal $i$ and $\mathbf{J}$ is a matrix of $1 \mathrm{~s}$ of order $\mathrm{T} \times \mathrm{T}$.

The RPG vector $\hat{\mathbf{a}}_{2}$ and corresponding $\hat{\gamma}$ have dimensions of $n \mathrm{~T} \times 1$ for the model with $\mathrm{T}$ traits; $\mathbf{B Z}$ in Equation [25] has dimensions of $m \mathrm{~T} \times n \mathrm{~T}$. The diagonal filter matrix $\mathbf{F}$ in Equation [24] becomes a blockdiagonal matrix for the $\mathrm{T}$ correlated traits with the size of $n \mathrm{~T} \times n \mathrm{~T}$. By extending Equation [25] to the multivariate model, SNP effects of $\mathrm{T}$ traits for all $m$ SNP markers are estimated by

$$
\hat{\mathbf{g}}=\left[\begin{array}{c}
\hat{\mathbf{g}}_{1} \\
\hat{\mathbf{g}}_{2} \\
\vdots \\
\hat{\mathbf{g}}_{m}
\end{array}\right]=\frac{1}{k}\left[\begin{array}{c}
\mathbf{B}_{11} \mathbf{Z}_{1}^{\prime} \\
\mathbf{B}_{22} \mathbf{Z}_{2}{ }^{\prime} \\
\vdots \\
\mathbf{B}_{m m} \mathbf{Z}_{m}^{\prime}
\end{array}\right] \mathbf{F} \hat{\gamma}
$$

In contrast to the SNP equations, the non-SNP equations of MME in Equation [16] can be straightforwardly extended to a multiple-trait model as in conventional evaluation.

\section{Frequent Genomic Evaluation Without New Phenotypes Added}

Unlike conventional genetic evaluation, genomic prediction is a more frequent process in dairy cattle; for example, once per week or month, as a result of continuous genotyping process. Most young candidates are genotyped between 2 conventional genetic evaluations where no new phenotypes are available for genomic prediction of those young candidates during the time period. To provide timely genomic evaluation, such as just-in-time instant genomic prediction, simple formulas need to be developed to avoid running the whole genomic evaluation process. Compared with the SSGblup models, our single-step SNP model [1] provides estimates of SNP effects; therefore, DGV of the genotyped young candidates can be easily obtained by summing all SNP effects. The $\mathbf{u}_{2}$ equation in [16] for young candidates that do not have their own phenotypes $\left(\mathbf{y}=\mathbf{0}, \mathbf{p}=\mathbf{0}, \mathbf{b}=\mathbf{0}\right.$, and $\left.\mathbf{W}_{2}=\mathbf{0}\right)$ becomes

$$
\mathbf{A}^{21} \hat{\mathbf{u}}_{1}+\mathbf{A}^{22} \hat{\mathbf{u}}_{2}+\left(\frac{1}{k}-1\right) \mathbf{A}_{22}^{-1} \hat{\mathbf{u}}_{2}=\frac{1}{k} \mathbf{A}_{22}^{-1} \mathbf{Z} \hat{\mathbf{g}} .
$$

Equation [36] can be rearranged as

$$
\mathbf{A}^{21} \hat{\mathbf{u}}_{1}+\mathbf{A}^{22} \hat{\mathbf{u}}_{2}-\mathbf{A}_{22}^{-1} \hat{\mathbf{u}}_{2}+\frac{1}{k} \mathbf{A}_{22}^{-1}\left(\hat{\mathbf{u}}_{2}-\mathbf{Z} \hat{\mathbf{g}}\right)=\mathbf{0} .
$$

Applying Equation [3], Equation [37] becomes

$$
\mathbf{A}^{21} \hat{\mathbf{u}}_{1}+\mathbf{A}^{22} \hat{\mathbf{u}}_{2}-\mathbf{A}_{22}^{-1}\left(\hat{\mathbf{u}}_{2}-\frac{1}{k} \hat{\mathbf{a}}_{2}\right)=\mathbf{0}
$$

For a candidate $l$ with sire $s$ and dam $d$, its diagonal in $\mathbf{A}^{22}$ is defined as $1+d_{l}$, under the assumption that the candidate has no progeny of its own:

$$
d_{l}=1 /\left[0.5-0.25\left(f_{s}+f_{d}\right)\right]
$$

where $f_{s}$ and $f_{d}$ are inbreeding coefficients of the sire $s$ and dam $d$, respectively. If a parent (e.g., dam) is unknown, $d_{l}=1 /\left(0.75-0.25 f_{s}\right)$; if neither parent is known, $d_{l}=1$ (Mrode, 2005). Similarly, the candidate's diagonal element in $\mathbf{A}_{22}^{-1}$ can be expressed in form $1+d_{l}^{*}$. The term $\mathbf{A}_{22}^{-1}\left(\hat{\mathbf{u}}_{2}-\frac{1}{k} \hat{\mathbf{a}}_{2}\right)$ in Equation [37] contains diagonal elements for the candidate $l$ as well as off-diagonal elements for all genotyped relatives of this candidate. For candidate $l$, the RPG effect is computed as follows:

$$
\hat{a}_{l}=\hat{u}_{l}-\mathbf{z}^{\prime} \hat{\mathbf{g}}
$$

where $\hat{u}_{l}$ is additive genetic effect of candidate $l$, and $\mathbf{z}$ is a vector of genotypes of candidate $l$. After rearranging some terms and applying Equation [39], Equation [38] for the candidate $l$ can be rewritten in scalar form:

$$
\begin{aligned}
& {\left[\frac{1}{k}\left(1+d_{l}^{*}\right)+\left(d_{l}-d_{l}^{*}\right)\right] \hat{u}_{l}-\frac{1}{2} d_{l}\left(\hat{u}_{s}+\hat{u}_{d}\right)-\frac{1}{k}\left(1+d_{l}^{*}\right) \mathbf{z}^{\prime} \hat{\mathbf{g}}} \\
& +\sum_{o=1}^{n_{o}} a_{l o}^{22}\left(\frac{1}{k} \hat{a}_{o}-\hat{u}_{o}\right)=0,
\end{aligned}
$$

where $\hat{u}_{s}$ and $\hat{u}_{d}$ are additive genetic effects of the sire $s$ and dam $d$, respectively, $n_{o}$ is number of genotyped relatives of candidate $l$ including its parents, $\hat{u}_{o}$ and $\hat{a}_{o}$ are additive genetic and RPG effects of relative $o$, and $a_{l o}^{22}$ is an off-diagonal element between candidate $l$ and its relative $o$ in $\mathbf{A}_{22}^{-1}$. The last summation term of Equation [40] describes contributions of genotyped relatives to the candidate $l$, which correspond to the row of candidate $l$ in the inverse relationship matrix $\mathbf{A}_{22}^{-1}$. Genotyped animals that are not related to the candidate $l$ are assumed here to make no contribution to $\hat{u}_{l}$ of the candidate as result of their off-diagonal elements with the candidate in $\mathbf{A}_{22}^{-1}$. From Equation [40], the additive genetic effect of the genotyped candidate $l$ can be derived as 


$$
\begin{aligned}
\hat{u}_{l}= & \frac{A}{A+B} \mathbf{z}^{\prime} \hat{\mathbf{g}}+\frac{d_{l}}{A+B} \frac{1}{2}\left(\hat{u}_{s}+\hat{u}_{d}\right) \\
& +\frac{1}{A+B} \sum_{o=1}^{n_{o}} a_{l o}^{22}\left(\hat{u}_{o}-\frac{1}{k} \hat{a}_{o}\right),
\end{aligned}
$$

where

$$
A=\frac{1}{k}\left(1+d_{l}^{*}\right) \text { and } B=d_{l}-d_{l}^{*} .
$$

Equation [41] shows that the additive genetic effect of candidate $l\left(\hat{u}_{l}\right)$ is a linear function of its $\operatorname{DGV}\left(\mathbf{z}^{\prime} \hat{\mathbf{g}}\right)$, parental average $\left[\frac{1}{2}\left(\hat{u}_{s}+\hat{u}_{d}\right)\right]$, and a correction term $\left(\hat{u}_{o}-\frac{1}{k} \hat{a}_{o}\right)$ for each of its genotyped relatives weighted by its off-diagonal element in $\mathbf{A}_{22}^{-1}$ with the relative $o$ $\left(a_{l o}^{22}\right)$. The third correction term in Equation [41] depends on the number of genotyped relatives and the off-diagonal elements $a_{l o}^{22}$ between the candidate $l$ and relative in $\mathbf{A}_{22}^{-1}$. Because $\hat{u}_{o}$ and $\frac{1}{k} \hat{a}_{o}$ have equal expected variance, $\hat{u}_{o}-\frac{1}{k} \hat{a}_{o}$, and thus the third term should not have large variance.

If all animals that are related to the candidate $l$ are genotyped, including, for example, both parents and grandparents, then Equation [41] can be simplified as a result of $d_{l}=d_{l}^{*}$ :

$$
\hat{u}_{l}=\mathbf{z}^{\prime} \hat{\mathbf{g}}+\frac{d_{l}}{1+d_{l}} \frac{1}{2}\left(\hat{a}_{s}+\hat{a}_{d}\right),
$$

where $\hat{a}_{s}$ or $\hat{a}_{d}$ represent RPG of the sire $s$ and dam $d$, respectively. If not all relatives are genotyped but both parents have genotypes available, then Equation [42] will be an approximation of Equation [41] because the third correction term in Equation [41] will not disappear; in this scenario, $d_{l}>d_{l}^{*}$ and $\mathrm{B}>0$. If dam $d$ is not genotyped but is known in pedigree, then again $\mathrm{B}>0$ due to $d_{l}>d_{l}^{*}$.

\section{DISCUSSION}

\section{The Single-Step SNP Model}

We proposed here an alternative single-step genomic model that directly estimates SNP effects and avoids explicitly defining the genomic relationship matrix $\mathbf{G}_{22}$ in contrast to the current single-step genomic model SSGblup (Aguilar et al., 2010; Christensen and Lund, 2010; Gao et al., 2012; Koivula et al., 2012; Su et al., 2012; Ødegård et al., 2013). We derived a simple and closed form of $\mathbf{H}^{-1}$ for additive genetic effects of nongenotyped animals, genotyped animals, and SNP ef- fects jointly. Because of the direct fitting of SNP effects in our single-step model [Eq. 1], there is no need to set up the genomic relationship matrix $\mathbf{G}_{22}$ and obtaining its inverse $\mathbf{G}_{22}^{-1}$, which we think is particularly important for national genomic evaluations involving hundreds of thousands of genotyped animals. However, the theoretical problem related to the definition of allele frequency from base or current populations identified in the GBLUP model (VanRaden, 2008) also exists here. Our single-step genomic model weights the observed genomic relationship and expected pedigree relationship implicitly using the parameter $k$, thus an extra step is not needed to weight both matrices, as in the single-step GBLUP models (Aguilar et al., 2010; Forni et al., 2011). Like all other single-step genomic models, our model requires calculating the matrix-vector products $\hat{\phi}=\mathbf{A}_{22}^{-1} \hat{\mathbf{u}}_{2}^{*}$ and $\hat{\gamma}=\mathbf{A}_{22}^{-1} \hat{\mathbf{a}}_{2}$, for which we successfully implemented the preconditioned conjugate gradients algorithm for German Holsteins that avoided not only inverting the matrix $\mathbf{A}_{22}$ but also storing the matrix $\mathbf{A}_{22}$ itself of all genotyped animals (Aguilar et al., 2011a). Consequently, our single-step model [Eq. 1] does not require storing or inverting either the pedigree relationship matrix $\mathbf{A}_{22}$ or the genomic relationship matrix G. Therefore, our model [Eq. 1] is suited perfectly to the iteration on data technique (Schaeffer and Kennedy, 1986) and thus feasible for populations of any size without a limit on the number of genotyped animals.

Our single-step genomic model [Eq. 1] allows flexible modeling of SNP effects in terms of the number and (co)variance structure of fitted SNP markers. If all SNP markers are assumed to contribute equal genetic variance, a BLUP SNP model (Liu et al., 2011) is fitted with (co)variance matrix $\mathbf{B}$ as shown in Equation [5]. A Bayesian model (Habier et al., 2011; Erbe et al., 2012) could be used to model the SNP effects but only if the individual SNP variances were known and stored in the diagonal matrix B. Our model also permits correlated SNP effects (Szyda et al., 2009). However, the number of correlated SNP must not be too high so that the matrix $\mathbf{B}$ can no longer be inverted in computer random access memory (RAM). Based on our model [Eq. 1], Goddard and Liu developed a one-step Bayesian model for genomic prediction (unpublished data). Because the matrix $\mathbf{B}$ and its inverse $\mathbf{B}^{-1}$ under the BLUP SNP or the Bayes SNP models have a very simple structure, in contrast to the genomic relationship matrix $\mathbf{G}_{22}$ of the SSGblup model, more efficient computing strategies can be developed by exploiting the structure of matrix B.

A distinct feature of our single-step SNP model [Eq. $1]$ is that the total genetic variance explained by all 
fitted SNP markers can be flexibly modeled using the parameter $k$, defined as the proportion of genetic variance not explained by all the fitted SNP markers. The parameter $k$ can take any value between 0 and 1 . If $k$ is very small (e.g., $k=0.0001$ ), the model [1] provides almost equivalent GEBV as a genomic model without a residual polygenic effect (see Appendix B for our single-step SNP model without RPG). Similarly, $k=$ 0.9999 would make our model [1] almost equivalent to a conventional genetic evaluation model without SNP marker effects. The RPG is important to reduce inflation of genomic prediction in practice. Parameter $k$ has mostly been estimated indirectly by performing genomic validation (Liu et al., 2011). Statistical methods are needed for optimally estimating parameter $k$. In most applications of the SSGblup model, the final genomic relationship matrix is typically calculated as a weighted average of the expected pedigree relationship matrix $\mathbf{A}_{22}$ and the observed genomic relationship matrix weighted by proportions of explained genetic variance (Christensen and Lund, 2010; Forni et al., 2011). Our single-step SNP model [Eq. 1] implicitly weighs both matrices using their associated proportions of genetic variance [Eq. 9]. This shows the analogy between our SNP model and the SSGblup model in handling the observed genomic and expected pedigree relationships.

Our single-step SNP model [Eq. 1] gives identical equations for additive genetic effects of genotyped and nongenotyped animals as the model by Gengler et al. (2012), although they derived their equations using a completely different approach via Quaas-Pollack transformation. In both models, a residual polygenic effect has been considered. If no RPG is assumed (Appendix B), then our model [1] provides identical equations for additive genetic effects as the SSGblup model by Legarra and Ducrocq (2012). However, one can see in Appendix B that both single-step models differ in the calculation of genomic contribution terms and SNP effect estimation.

\section{SNP Effect Estimation}

We developed a new algorithm to estimate SNP effects by converting estimated additive genetic effects [Eq. 17]. By exploring the relationship of DGV and RPG between 2 consecutive rounds of iteration [Eq. 26], we developed an efficient procedure for updating RPG. This updating procedure resembles the GaussSeidel solving algorithm with a special residual update by Legarra and Misztal (2008). Our special updating algorithm can reduce computing operations and thus make the computing time increase approximately linearly with the number of genotyped animals. We were concerned that a very small value of the proportion of residual polygenic variance $(k)$ might lead to poorer convergence. According to experience with our current BLUP SNP model including a residual polygenic effect (Liu et al., 2011), $k$ values actually ranged from 0.0001 to 0.5 between traits in routine or test evaluations, and no difference in convergence criteria has been observed among traits with different $k$ values.

In contrast to the SSGblup model, our single-step SNP model actively controls the information flow from genomic reference population to candidates by allowing only selected reference animals to contribute to the conversion of additive genetic to SNP effects [Eq. 25]. The filter matrix $\mathbf{F}$ excludes some genotyped animals; for example, bull dams with potentially biased additive genetic effects or bulls with very limited daughter information from the genomic reference population, so that the estimated SNP effects will not be influenced by those problematic genotyped animals. In routine genomic evaluations of German Holsteins this type of selection of reference animals has been proven to be extremely important to guarantee unbiased genomic prediction (unpublished data). The extra step of estimating SNP effects in our single-step SNP model, in contrast to SSGblup model, makes the selection of reference animals possible. However, it is important to note that the selection of reference animals in SNP effect estimation will not lead to biased GEBV as the case of genomic preselection (Patry and Ducrocq, 2011), because all genotyped animals, including culled candidates, are considered in Equation [16] even though only some of them are qualified to be included in genomic reference population.

In a multi-step genomic model such as Liu et al. (2011), the SNP effects are estimated using deregressed EBV after conventional genetic evaluation. In contrast, SNP effect estimation of our single-step SNP model directly converts estimates of additive genetic effects to SNP effects [Eq. 25]. The intermediate step of deregressing EBV is no longer required in the single-step SNP model, at least for bulls included in national conventional evaluations. Therefore, extreme DRP values for bulls or cows with very little phenotypic data should become less problematic in SNP effect estimation or genomic evaluation.

\section{An Extension to Multiple Traits}

Our single-step SNP model [Eq. 1] can be readily extended to analyze multiple traits jointly. The extension for additive genetic effects in Equation [16] can be done in the same way as in conventional genetic evaluation. For SNP effect estimation [Eq. 25], the SNP (co)variance matrix $\mathbf{B}$ becomes block-diagonal instead of diagonal. Because of the simple structure of block- 
diagonal B matrix, multiple-trait SNP effects can also be estimated efficiently.

Correlations of the same SNP marker between traits on an SNP level [Eq. 30] are assumed to be equal to the correlations of additive genetic effects on the genome level [Eq. 28]. Ideally, estimated correlations of traits on the SNP level could be used here. However, it is extremely difficult to accurately estimate those correlations between traits on the SNP level due to a large number of autocorrelated SNP markers. For a BLUP SNP model (Liu et al., 2011), the assumption of equal trait correlations on both the SNP and genome levels can be easily satisfied, if equal proportion of residual polygenic variance $k$ is assumed across all the correlated traits. However, for Bayesian SNP models assuming heterogeneous SNP variances for the different traits, it is no longer straightforward to derive correlations of traits on the SNP level from those on the genome level. This makes the extension to multiple traits more difficult for the Bayesian than for the BLUP SNP models.

In SSGblup model applications, the observed genomic relationship matrix is usually combined with the expected pedigree relationship matrix to reduce inflation of genomic prediction; the resulting weighted genomic relationship matrix is then applied to all traits in a multiple-trait genomic evaluation (Aguilar et al., 2011b; Christensen et al., 2012). In this case, the SSGblup model makes an implicit assumption of equal weights across all the correlated traits. Likewise, our single-step SNP model with multiple correlated traits applies the same parameter $k$ for the proportion of residual polygenic variance to all the traits under a BLUP SNP model like that of Liu et al. (2011). This assumption of equal proportion of residual polygenic variance across all correlated traits in a multi-trait evaluation has been proven to be realistic for most trait groups in dairy cattle, such as milk production traits, female fertility traits, calving traits, and so on. However, this assumption will become less realistic for conformation traits covering a wider range of variation in the proportion of residual polygenic variance. Technical solutions need to be developed to get around this assumption.

\section{Computing Strategies}

Equations of our single-step SNP model [Eq. 1] consist of conventional and pure genomic parts. Besides the additional step of SNP effect estimation [Eq. 25], we can see from Equation [18] that only the RHS of genotyped animals needs to be adjusted for the genomic contribution compared with MME of conventional evaluations. The calculation of the genomic contribution involves 2 multiplications, $\hat{\phi}=\mathbf{A}_{22}^{-1} \hat{\mathbf{u}}_{2}^{*}$ and $\hat{\gamma}=\mathbf{A}_{22}^{-1} \hat{\mathbf{a}}_{2}$, for which numerical computing algorithms such as preconditioned conjugate gradients can be implemented. Computing strategies for solving the conventional part of MME [Eq. 16] can be implemented, such as iteration on data technique (Schaeffer and Kennedy, 1986) or preconditioned conjugate gradient algorithm (Strandén and Lidauer, 1999). For the SNP effect estimation [Eq. 25], the genotype file needs to be read only once per round of iteration [Eq. 26] plus the extra calculation $\hat{\gamma}=\mathbf{A}_{22}^{-1} \hat{\mathbf{a}}_{2}$. The single-step SNP model allows us to take advantage of the simple structure of diagonal or block-diagonal matrix $\mathbf{B}$ to make the SNP effect estimation efficient.

It is a computational challenge to get all the effects of the single-step SNP model [1] properly converged and accurately estimated, because we have to estimate not only all the effects included in a conventional genetic evaluation; for example, a random regression testday model for milk yield (Liu et al., 2004), but also the SNP marker effects such as those in a multi-step BLUP SNP model (VanRaden, 2008; Liu et al., 2011; Lund et al., 2011). Combining the conventional and genomic evaluations into a single evaluation system requires special computing strategies to guarantee convergence of the whole equation system and to achieve a reasonable accuracy of the estimated effects within the allowed time frame in routine genetic evaluation. For example, the German Holstein population includes approximately 20 million cows having test-day data and more than 100,000 genotyped animals. Although the 2 components, conventional and genomic evaluations, have been proven to be converged fairly well in routine conventional evaluations or genomic evaluations with a multi-step genomic model, the combined equation system [Eq. 15] of the single-step SNP model might have a slower rate of convergence rate than either of its components, because these 2 subsets of equations, Equation [16] for the non-SNP effects and Equation [17] for SNP effects, are related. The separation of these 2 subsets of equations allows us to flexibly apply different intensity of iteration on the effects in the 2 subsets of equations. For example, we may perform many rounds of iteration on Equation [16] at the beginning of estimation process with priors of SNP effects to achieve a reasonable accuracy for all effects in Equation [16] before estimating the SNP effects with Equation [17]. However, the block iterative solving of Equations [16] and [17] may need more computations than solving Equation [15] as a whole, because the complete equation system [Eq. 15] may be more tolerant to inaccurate interim solutions of the model effects than the 2 separate sets of equations. In contrast to Equation [16], all the genomic parts are moved to the RHS of Equation [18], making the lefthand side of Equation [18] identical as that of conven- 
tional genetic evaluation. This rearrangement of the model terms allows us to use existing, usually complex, computing software from conventional genetic evaluation for implementing the single-step genomic model. However, the rearranged Equation [18] may require more rounds of iteration to achieve convergence than the original Equation [16]. Compared with the model by Legarra and Ducrocq (2012), our single-step model [Eq. 1] estimates SNP effects by converting additive genetic effects $\mathbf{u}_{2}$ with matrix $\mathbf{A}_{22}^{-1}$ (Equation [21]), whereas their model estimates SNP effects using the inverse of genomic relationship matrix $\mathbf{G}_{22}^{-1}$ (Equation [B8] in Appendix B). Although these 2 models are shown to be equivalent for estimating the SNP effects (see Appendix B), our model explicitly divides the additive genetic effects $\mathbf{u}_{2}$ into SNP marker effects $\mathbf{g}$ and RPG $\mathbf{a}_{2}$ by assuming the proportion $(k)$ of residual polygenic variance in the total additive genetic variance. An analogy can be found in their single-step GBLUP model in weighting the observed genomic relationship with pedigree relationship matrix $\mathbf{A}_{22}$. The convergence rate of our single-step SNP model [1] needs to be compared with the single-step GBLUP model by Legarra and Ducrocq (2012). Although we have not found any effect of different $k$ values on convergence criteria in our current multi-step genomic model (Liu et al., 2011), it is important to investigate the influence of the parameter $k$ on the rate of convergence and the tolerance of parameter $k$ on interim solutions in our single-step SNP model [Eq. 1]. The SNP effects $\mathrm{g}$ appear on both sides of Equation [21]; therefore, iteration between Equations [26] and [27] is needed to properly separate $\mathbf{u}_{2}$ into SNP effects $\mathbf{g}$ and residual polygenic effects $\mathbf{a}_{2}$. In fact, this special estimation procedure worked efficiently in a multi-step BLUP SNP model for German Holsteins (Liu et al., 2011).

In summary, jointly estimating all the effects of our single-step SNP model [Eq. 1] is a challenging and difficult computational task for routine evaluations in Holsteins with hundreds of thousands of genotyped animals and tens of millions of cows with phenotypes. The original complete set of Equation [15] has been modified considerably to make use of existing software for current conventional evaluations and to allow flexible iteration on the 2 subsets of equations. Further research is required to investigate the effects of different values of parameter $k$ and levels of trait heritability on convergence behavior of the single-step SNP model. Past implementation experience from conventional genetic and multi-step genomic evaluations can be learned to make the single-step SNP model work correctly and efficiently for routine evaluations with very large data sets of phenotypes and genotypes.

\section{Frequent Genomic Prediction}

Because of continuous genotyping of candidates in Holstein breeding programs, breeders want to know results of genomic evaluation as soon as genotypes have been made available for an immediate culling decision. Therefore, we developed simple formulas [Eq. 41 and 42] for an instant, "just-in-time" genomic evaluation without running the whole process of genomic evaluation. These simple formulas for genomic prediction work correctly only when no new phenotypes are added after a complete genetic evaluation. Under such conditions, SNP effect estimates from the previous genomic evaluation can be used as well as GEBV of genotyped and EBV of nongenotyped animals from the previous evaluation. The prediction formula [41] is only valid for genotyped candidates without their own phenotypes, and it can also be used to explain relative contributions of 3 components for GEBV of the candidates: own genotype, parental contribution, and genomic contribution of other genotyped relatives. Because genomic prediction can be quickly made available for genotyped candidates using the above formulas, breeding organizations or dairy cattle breeders can make selection decision immediately and reduce costs of genomic selection.

\section{Effect of Genomic Preselection}

The most important superiority of single-step models over the current multi-step genomic models is that conventional genetic evaluation and following genomic prediction will not be biased in the presence of genomic preselection (Patry and Ducrocq, 2011). Like the SSGblup model (Aguilar et al., 2010), our single-step SNP model considers all genotyped animals, including culled animals. The influence of a culled candidate on its parents can be seen in Equation [40]. As shown in Equation [25], only selected animals are allowed to contribute the SNP effect estimation to reduce the problem of less accurate or biased GEBV of certain groups of animals on the SNP effect estimates. But the selection of the genomic reference animals in the conversion of GEBV to SNP effects should not lead to biased genomic prediction as the case of the multi-step genomic model in presence of genomic preselection.

\section{Implementation Issues}

Implementing the single-step SNP model [Eq. 1] requires many technical steps; in particular, for example, in German Holsteins with an across-country genomic reference population (Lund et al., 2011). Because the majority of EuroGenomics across-country reference bulls do not have daughters in Germany, MACE EBV 
of those foreign bulls must be deregressed as phenotypes of the genomic evaluation (Liu, 2011). Animals contributing phenotypic data for the single-step genomic evaluation of, for example, milk production traits, are domestic cows with test-day records (Liu et al., 2004) and foreign bulls with MACE deregressed EBV. Because MACE evaluation is based on a single-trait model (e.g., milk yield of combined first 3 lactations on 305-d basis), a difference exists in trait definitions between foreign bulls and domestic cows with test-day yields evaluated with 3 Legendre polynomial coefficients in each of first 3 lactations (Liu et al., 2004). The 2 definitions of the same trait need to be converted within the single-step genomic model.

In the current genomic evaluation for German Holsteins (Liu et al., 2011) based on a multi-step genomic model, EBV of dam are not directly used in the calculation of pedigree index for candidates for fear of overestimating EBV of elite cows or dams of bulls. Instead, only EBV of bulls are used in the pedigree index calculation. This strategy has been proven effective in German Holsteins to remove the effect of overestimated bull dams, particularly for milk production traits (unpublished data). In order for the single-step SNP model [Eq. 1] to avoid bias from overestimated cow EBV, dams of candidates with phenotypes may need to receive special adjustments for possible overestimation of EBV. By doing so, the overestimation problem of elite cows will not be passed to the next-generation candidates.

Reliabilities of GEBV of the single-step SNP model [Eq. 1] have to be approximated, possibly following a procedure similar to that in a conventional evaluation using a random regression test-day model (Liu et al., 2004). Pure genomic contribution may be treated as a new source of information, besides the contribution by own data and parental and progeny contributions, as described in a multi-trait effective daughter contribution method (Liu et al., 2004). However, calculating the pure genomic contribution, which should be independent of the other 3 sources of information, is technically very challenging, because this involves the inverse of $\mathbf{G}_{22}^{-1}-\mathbf{A}_{22}^{-1}$. For the Holstein breed with an acrosscountry genomic reference population, the number of genotyped animals is too large to invert the matrices or even to set up the matrices in computer memory. Approximation of the inverse of $\mathbf{G}_{22}^{-1}-\mathbf{A}_{22}^{-1}$ has to be done instead. Possible overestimation of genomic reliability by using the approximated inverse may be adjusted via a genomic validation study. Research is needed to develop statistical methods for unbiased reliabilities for the single-step SNP model [Eq. 1].

\section{CONCLUSIONS}

We developed a single-step genomic model that allows direct estimation of SNP effects. The single-step SNP model does not require setting up either the observed genomic or the expected pedigree relationship matrix or inverting those matrices for genotyped animals, which makes the model applicable for any number of genotyped animals. For computing genomic contributions (i.e., $\mathbf{A}_{22}^{-1} \hat{\mathbf{u}}_{2}^{*}$ or $\mathbf{A}_{22}^{-1} \hat{\mathbf{a}}_{2}$ ), we successfully implemented and validated the preconditioned conjugate gradients algorithm in German Holsteins. The SNP effects can be estimated using a special algorithm that separates SNP effects efficiently from residual polygenic effects. Additionally, we extended our single-step SNP model to general multiple-trait evaluations. Because of the block-diagonal (co)variance matrix B for multivariate SNP effects, SNP effect estimation is straightforward for the multi-trait single-step SNP model. For frequent interim genomic evaluations without new phenotypes added, we derived simple prediction formulas for GEBV of candidates without running the whole genomic evaluation process. Several aspects related to the implementation of the model were discussed: effect of genomic preselection, adjusting overestimated EBV of elite cows or bull dams, genomic evaluation using an across-country reference population and international phenotypes, and reliability approximation for the single-step SNP model.

\section{ACKNOWLEDGMENTS}

We kindly thank Paul VanRaden (USDA Animal Improvement Programs Laboratory, Beltsville, MD) and Vincent Ducrocq (INRA, Jouy-en-Josas, France) for discussing the calculation of $\mathbf{A}_{22}^{-1} \hat{\mathbf{u}}_{2}^{*}$. Nicolas Gengler (University of Liège, Gembloux, Belgium) and Andres Legarra (INRA, Castanet Tolosan, France) are acknowledged for providing useful discussion about our single-step SNP genomic model. We appreciated very much their competent review and suggestions by two anonymous reviewers that improved our manuscript.

\section{REFERENCES}

Aguilar, I., I. Misztal, D. L. Johnson, A. Legarra, S. Tsuruta, and T. J. Lawlor. 2010. Hot topic: A unified approach to utilize phenotypic, full pedigree, and genomic information for genetic evaluation of Holstein final score. J. Dairy Sci. 93:743-752.

Aguilar, I., I. Misztal, A. Legarra, and S. Tsuruta. 2011a. Efficient computations of genomic relationship matrix and other matrices used in the single-step evaluation. J. Anim. Breed. Genet. 128:422-428.

Aguilar, I., I. Misztal, S. Tsuruta, G. R. Wiggans, and T. J. Lawlor. 2011b. Multiple trait genomic evaluation of conception rate in Holsteins. J. Dairy Sci. 94:2621-2624. 
Calus, M. P., and R. F. Veerkamp. 2011. Accuracy of multi-trait genomic selection using different methods. Genet. Sel. Evol. 43:26.

Christensen, O. F., and M. S. Lund. 2010. Genomic prediction when some animals are not genotyped. Genet. Sel. Evol. 42:2.

Christensen, O. F., P. Madsen, B. Nielsen, T. Ostersen, and G. Su. 2012. Single-step methods for genomic evaluation in pigs. Animal $6: 1565-1571$

Erbe, M., B. J. Hayes, L. K. Matukumali, S. Goswami, P. J. Bowman, C. M. Reich, B. A. Mason, and M. E. Goddard. 2012. Improving accuracy of genomic predictions within and between dairy cattle breeds with imputed high-density single nucleotide polymorphism panels. J. Dairy Sci. 95:4114-4129.

Forni, S., I. Aguilar, and I. Misztal. 2011. Different genomic relationship matrices for single-step analysis using phenotypic, pedigree and genomic information. Genet. Sel. Evol. 43:1. http://dx.doi. org/10.1186/1297-9686-43-1.

Gao, H., O. F. Christensen, P. Madsen, U. S. Nielsen, Y. Zhang, M. S. Lund, and G. Su. 2012. Comparison on genomic predictions using three GBLUP methods and two single-step blending methods in the Nordic Holstein population. Genet. Sel. Evol. 44:8. http:// dx.doi.org/10.1186/1297-9686-44-8.

Gengler, N., G. Nieuwhof, K. Konstantinov, and M. Goddard. 2012. Alternative single-step type genomic prediction equations. Book of Abstracts 18:131 in Proc. 63rd Annu. Mtg. Eur. Fed. Anim. Sci., Bratislava, Slovakia. Wageningen Academic Publishers, Wageningen, the Netherlands.

Habier, D., R. L. Fernando, K. Kizilkaya, and D. Garrick. 2011. Extension of the Bayesian alphabet for genomic selection. BMC Bioinformatics $12: 186$.

Harville, D. A. 1997. Matrix Algebra from a Statistician's Perspective. Springer-Verlag, New York, NY.

Jairath, L., J. C. M. Dekkers, L. R. Schaeffer, Z. Liu, E. B. Burnside, and B. Kolstad. 1998. Genetic evaluation for herd life in Canada. J. Dairy Sci. 81:550-562.

Koivula, M., I. Strandén, G. Su, and E. A. Mäntysaari. 2012. Different methods to calculate genomic predictions - Comparisons of BLUP at the single nucleotide polymorphism level, BLUP at the individual level (G-BLUP), and the one-step approach (H-BLUP). J. Dairy Sci. 95:4065-4073.

Legarra, A., and V. Ducrocq. 2012. Computational strategies for national integration of phenotypic, genomic, and pedigree data in a single-step best linear unbiased prediction. J. Dairy Sci. 95:46294645 .

Legarra, A., and I. Misztal. 2008. Technical note: Computing strategies in genome-wide selection. J. Dairy Sci. 91:360-366.

Liu, Z. 2011. Use of MACE results as input for genomic models. Interbull Bull. 43:1-4.

Liu, Z., F. Reinhardt, A. Bünger, and R. Reents. 2004. Derivation and calculation of approximate reliabilities and daughter yield deviations of a random regression test-day model for genetic evaluation of dairy cattle. J. Dairy Sci. 87:1896-1907.

Liu, Z., F. R. Seefried, R. Reinhardt, S. Rensing, G. Thaller, and R. Reents. 2011. Impacts of both reference population size and inclusion of a residual polygenic effect on the accuracy of genomic prediction. Genet. Sel. Evol. 43:19. http://dx.doi.org/10.1186/12979686-43-19.

Lund, M. S., A. P. W. De Roos, A. G. De Vries, T. Druet, V. Ducrocq, S. Fritz, F. Guillaume, B. Guldbrandtsen, Z. Liu, R. Reents, C. Schrooten, F. Seefried, and G. Su. 2011. A common reference population from four European Holstein populations increases reliability of genomic predictions. Genet. Sel. Evol. 43:43.http:// dx.doi.org/10.1186/1297-9686-43-19.

Meuwissen, T. H. E., B. J. Hayes, and M. E. Goddard. 2001. Prediction of total genetic value using genome-wide dense marker maps. Genetics 157:1819-1829.

Misztal, I., A. Legarra, and I. Aguilar. 2009. Computing procedures for genetic evaluation including phenotypic, full pedigree, and genomic information. J. Dairy Sci. 92:4648-4655.

Mrode, R. A. 2005. Linear Models for the Prediction of Animal Breeding Values. 2nd ed. CAB International, Wallingford, UK.
Ødegård, J., P. Madsen, and T. H. E. Meuwissen. 2013. An inversion-free method to compute genomic predictions using an animal model approach. Book of Abstracts 19:454 in Proc. 64th Annu. Mtg. Eur. Fed. Anim. Sci., Nantes, France. Wageningen Academic Publishers, Wageningen, the Netherlands.

Patry, C., and V. Ducrocq. 2011. Evidence of biases in genetic evaluations due to genomic preselection in dairy cattle. J. Dairy Sci. 94:1011-1020.

Schaeffer, L. R. 1994. Multiple-country comparison of dairy sires. J. Dairy Sci. 77:2671-2678.

Schaeffer, L. R., and B. W. Kennedy. 1986. Computing strategies for solving mixed model equations. J. Dairy Sci. 69:575-579.

Strandén, I., and D. J. Garrick. 2009. Technical note: Derivation of equivalent computing algorithms for genomic predictions and reliabilities of animal merit. J. Dairy Sci. 92:2971-2975.

Strandén, I., and M. Lidauer. 1999. Solving large mixed linear models using preconditioned conjugate gradient iteration. J. Dairy Sci. 82:2779-2787.

Su, G., P. Madsen, U. S. Nielsen, E. A. Mäntysaari, G. P. Aamand, O. F. Christensen, and M. S. Lund. 2012. Genomic prediction for Nordic Red cattle using one-step and selection index blending. J. Dairy Sci. 95:909-917.

Szyda, J., S. Kaminski, A. Zarnecki, and K. Zukowski. 2009. Incorporation of correlation between SNPs into genomic evaluation model. Interbull Bull. 40:193-197.

VanRaden, P. M. 2008. Efficient methods to compute genomic predictions. J. Dairy Sci. 91:4414-4423.

VanRaden, P. M., C. P. Van Tassell, G. R. Wiggans, T. S. Sonstegard, R. D. Schnabel, J. F. Taylor, and F. S. Schenkel. 2009. Invited review: Reliability of genomic predictions for North American Holstein bulls. J. Dairy Sci. 92:16-24.

\section{APPENDIX A: DERIVATION OF THE INVERSE MATRICES OF G AND H}

We show here derivations of the inverse of matrices $\mathbf{G}$ and $\mathbf{H}$, which are given in Equations [11] and [13], respectively. To derive the inverse matrices, we need to express additive genetic effects of nongenotyped animals $\left(\mathbf{u}_{1}\right)$ in terms of those of genotyped animals $\left(\mathbf{u}_{2}\right)$ :

$$
\mathbf{u}_{1}=\mathbf{T} \mathbf{u}_{2}+\mathbf{d},
$$

where $\mathbf{T}=\mathbf{A}_{12} \mathbf{A}_{22}^{-1}$ is a transmission matrix connecting genotyped to nongenotyped animals, and $\mathbf{d}$ is a vector of deviation effects with a (co)variance matrix

$$
\operatorname{var}(\mathbf{d})=\mathbf{D} \sigma_{u}^{2} .
$$

Further, it is assumed that the deviation effects $\mathbf{d}$ of the nongenotyped animals have a zero covariance with $\mathbf{u}_{2}$ of the genotyped animals, $\operatorname{cov}\left(\mathbf{u}_{2}, \mathbf{d}\right)=\mathbf{0}$. It can be shown that

$$
\mathbf{D}^{-1}=\mathbf{A}^{11} \text {. }
$$

We give a proof of Equation [A3] at the end of this Appendix A. The transmission matrix $\mathbf{T}$ may be extremely large. However, we introduce the transmission matrix $\mathbf{T}$ only for the purpose of deriving the inverse matrices of $\mathbf{G}$ and $\mathbf{H}$, which no longer contain $\mathbf{T}$. 
(Co)variance matrix of additive genetic effects of both groups of animals (Equation [10]) is by applying [A1]:

$$
\operatorname{var}\left(\begin{array}{l}
\mathbf{u}_{1} \\
\mathbf{u}_{2}
\end{array}\right)=\mathbf{G} \sigma_{u}^{2}=\left[\begin{array}{cc}
\mathbf{T G}_{22} \mathbf{T}^{\prime}+\mathbf{D} & \mathbf{T G}_{22} \\
\mathbf{G}_{22} \mathbf{T}^{\prime} & \mathbf{G}_{22}
\end{array}\right] \sigma_{u}^{2}
$$

and inverse of matrix $\mathbf{G}$ can be obtained algebraically:

$$
\begin{aligned}
\mathbf{G}^{-1} & =\left[\begin{array}{cc}
\mathbf{D}^{-1} & -\mathbf{D}^{-1} \mathbf{T} \\
-\mathbf{T}^{\prime} \mathbf{D}^{-1} & \mathbf{G}_{22}^{-1}+\mathbf{T}^{\prime} \mathbf{D}^{-1} \mathbf{T}
\end{array}\right] \\
& =\left[\begin{array}{cc}
\mathbf{A}^{11} & \mathbf{A}^{12} \\
\mathbf{A}^{21} & \mathbf{G}_{22}^{-1}+\mathbf{A}^{22}-\mathbf{A}_{22}^{-1}
\end{array}\right] .
\end{aligned}
$$

Equation [A6] for $\mathbf{G}^{-1}$ is equal to $\mathbf{H}^{-1}$ by Aguilar et al. (2010) and $\left(\tilde{G}_{w}\right)^{-1}$ by Christensen and Lund (2010). Below we show our proof that multiplying $\mathbf{G}$ in [A4] with its inverse $\mathbf{G}^{-1}$ in Equation [A5] gives an identity matrix:

$$
\begin{aligned}
\mathbf{G G}^{-1} & =\left[\begin{array}{cc}
\mathbf{T G}_{22} \mathbf{T}^{\prime}+\mathbf{D} & \mathbf{T G}_{22} \\
\mathbf{G}_{22} \mathbf{T}^{\prime} & \mathbf{G}_{22}
\end{array}\right]\left[\begin{array}{cc}
\mathbf{D}^{-1} & -\mathbf{D}^{-1} \mathbf{T} \\
-\mathbf{T}^{\prime} \mathbf{D}^{-1} & \mathbf{G}_{22}^{-1}+\mathbf{T}^{\prime} \mathbf{D}^{-1} \mathbf{T}
\end{array}\right] \\
& =\left[\begin{array}{ll}
\mathbf{P}_{11} & \mathbf{P}_{12} \\
\mathbf{P}_{21} & \mathbf{P}_{22}
\end{array}\right]=\left[\begin{array}{ll}
\mathbf{I} & \mathbf{0} \\
\mathbf{0} & \mathbf{I}
\end{array}\right],
\end{aligned}
$$$$
\mathbf{P}_{11}=\left(\mathbf{T G}_{22} \mathbf{T}^{\prime}+\mathbf{D}\right) \mathbf{D}^{-1}-\mathbf{T G}_{22} \mathbf{T}^{\prime} \mathbf{D}^{-1}
$$$$
=\mathbf{T G}_{22} \mathbf{T}^{\prime} \mathbf{D}^{-1}+\mathbf{I}-\mathbf{T G}_{22} \mathbf{T}^{\prime} \mathbf{D}^{-1}=\mathbf{I},
$$$$
\mathbf{P}_{12}=-\left(\mathbf{T G}_{22} \mathbf{T}^{\prime}+\mathbf{D}\right) \mathbf{D}^{-1} \mathbf{T}+\mathbf{T G}_{22}\left(\mathbf{G}_{22}^{-1}+\mathbf{T}^{\prime} \mathbf{D}^{-1} \mathbf{T}\right)
$$$$
=-\mathbf{T G}_{22} \mathbf{T}^{\prime} \mathbf{D}^{-1} \mathbf{T}-\mathbf{T}+\mathbf{T}+\mathbf{T G}_{22} \mathbf{T}^{\prime} \mathbf{D}^{-1} \mathbf{T}=\mathbf{0},
$$$$
\mathbf{P}_{21}=\mathbf{G}_{22} \mathbf{T}^{\prime} \mathbf{D}^{-1}-\mathbf{G}_{22} \mathbf{T}^{\prime} \mathbf{D}^{-1}=\mathbf{0},
$$$$
\mathbf{P}_{22}=-\mathbf{G}_{22} \mathbf{T}^{\prime} \mathbf{D}^{-1} \mathbf{T}+\mathbf{G}_{22}\left(\mathbf{G}_{22}^{-1}+\mathbf{T}^{\prime} \mathbf{D}^{-1} \mathbf{T}\right)
$$$$
=\mathbf{I}-\mathbf{G}_{22} \mathbf{T}^{\prime} \mathbf{D}^{-1} \mathbf{T}+\mathbf{G}_{22} \mathbf{T}^{\prime} \mathbf{D}^{-1} \mathbf{T}=\mathbf{I} \text {. }
$$

To prove the equality of Equations [A5] and [A6] for $\mathbf{G}^{-1}$, we need some matrix inversion rules (Harville, 1997) for the partitioned relationship matrix:

$$
\left[\begin{array}{ll}
\mathbf{A}_{11} & \mathbf{A}_{12} \\
\mathbf{A}_{21} & \mathbf{A}_{22}
\end{array}\right]^{-1}=\left[\begin{array}{ll}
\mathbf{A}^{11} & \mathbf{A}^{12} \\
\mathbf{A}^{21} & \mathbf{A}^{22}
\end{array}\right]
$$

According to the rules in Harville (1997; pages 98-101), the following relationships exist:

$$
\begin{aligned}
\mathbf{A}^{21} & =-\mathbf{A}_{22}^{-1} \mathbf{A}_{21} \mathbf{A}^{11} \text { and } \\
\mathbf{A}^{22} & =\mathbf{A}_{22}^{-1}-\mathbf{A}^{21} \mathbf{A}_{12} \mathbf{A}_{22}^{-1} .
\end{aligned}
$$

Replacing $\mathbf{A}^{21}$ in Equation [A10] using Equation [A9] results in

$$
\mathbf{A}^{22}-\mathbf{A}_{22}^{-1}=\mathbf{A}_{22}^{-1} \mathbf{A}_{21} \mathbf{A}^{11} \mathbf{A}_{12} \mathbf{A}_{22}^{-1} .
$$

The off-diagonal elements of $\mathbf{G}^{-1}$ in Equation [A5] are thus

$$
\begin{gathered}
-\mathbf{T}^{\prime} \mathbf{D}^{-1}=-\mathbf{A}_{22}^{-1} \mathbf{A}_{21} \mathbf{A}^{11}=\mathbf{A}^{21} \text { and } \\
-\mathbf{D}^{-1} \mathbf{T}=-\left(\mathbf{T}^{\prime} \mathbf{D}^{-1}\right)^{\prime}=-\left(-\mathbf{A}^{21}\right)^{\prime}=\mathbf{A}^{12} .
\end{gathered}
$$

Applying Equation [A11], the term $\mathbf{T}^{\prime} \mathbf{D}^{-1} \mathbf{T}$ of the second diagonal element of $\mathbf{G}^{-1}$ in Equation [A5] is

$$
\mathbf{T}^{\prime} \mathbf{D}^{-1} \mathbf{T}=\mathbf{A}_{22}^{-1} \mathbf{A}_{21} \mathbf{A}^{11} \mathbf{A}_{12} \mathbf{A}_{22}^{-1}=\mathbf{A}^{22}-\mathbf{A}_{22}^{-1} .
$$

The equality of Equations [A5] and [A6] for $\mathbf{G}^{-1}$ has thus been proven.

In contrast to the single-step GBLUP models (Aguilar et al., 2010; Christensen and Lund, 2010; Legarra and Ducrocq, 2012), our single-step genomic model adds a vector of SNP effects $\mathbf{g}$ to $\mathbf{u}$, forming the vector $\mathbf{h}$ that has a (co)variance matrix (Equation [12])

$$
\operatorname{var}\left[\begin{array}{c}
\mathbf{u}_{1} \\
\mathbf{u}_{2} \\
\mathbf{g}
\end{array}\right]=\mathbf{H} \sigma_{u}^{2}=\left[\begin{array}{ccc}
\mathbf{T G}_{22} \mathbf{T}^{\prime}+\mathbf{D} & \mathbf{T G}_{22} & \mathbf{T Z B} \\
\mathbf{G}_{22} \mathbf{T}^{\prime} & \mathbf{G}_{22} & \mathbf{Z B} \\
\mathbf{B Z} \mathbf{T}^{\prime} & \mathbf{B Z} & \mathbf{B}
\end{array}\right] \sigma_{u}^{2},
$$

and inverse of matrix $\mathbf{H}$

$$
\begin{aligned}
\mathbf{H}^{-1} & =\left[\begin{array}{ccc}
\mathbf{D}^{-1} & -\mathbf{D}^{-1} \mathbf{T} & \mathbf{0} \\
-\mathbf{T}^{\prime} \mathbf{D}^{-1} & \frac{1}{k} \mathbf{A}_{22}^{-1}+\mathbf{T}^{\prime} \mathbf{D}^{-1} \mathbf{T} & -\frac{1}{k} \mathbf{A}_{22}^{-1} \mathbf{Z} \\
\mathbf{0} & -\frac{1}{k} \mathbf{Z}^{\prime} \mathbf{A}_{22}^{-1} & \mathbf{B}^{-1}+\frac{1}{k} \mathbf{Z}^{\prime} \mathbf{A}_{22}^{-1} \mathbf{Z}
\end{array}\right] \\
& =\left[\begin{array}{ccc}
\mathbf{A}^{11} & \mathbf{A}^{12} & \mathbf{0} \\
\mathbf{A}^{21} & \mathbf{A}^{22}+\left(\frac{1}{k}-1\right) \mathbf{A}_{22}^{-1} & -\frac{1}{k} \mathbf{A}_{22}^{-1} \mathbf{Z} \\
\mathbf{0} & -\frac{1}{k} \mathbf{Z}^{\prime} \mathbf{A}_{22}^{-1} & \mathbf{B}^{-1}+\frac{1}{k} \mathbf{Z}^{\prime} \mathbf{A}_{22}^{-1} \mathbf{Z}
\end{array}\right] \cdot[\mathrm{A}
\end{aligned}
$$


Equality of Equations [A16] and [A17] can be seen from the above verification showing Equation [A5] is equal to Equation [A6]. To prove Equation [A16] for $\mathbf{H}^{-1}$, we give here our verification demonstrating that multiplying Equation [A15] for $\mathbf{H}$ with Equation [A16] for $\mathbf{H}^{-1}$ gives an identity matrix:

$$
\begin{aligned}
& \mathbf{H H}^{-1}=\left[\begin{array}{lll}
\mathbf{Q}_{11} & \mathbf{Q}_{12} & \mathbf{Q}_{13} \\
\mathbf{Q}_{21} & \mathbf{Q}_{22} & \mathbf{Q}_{23} \\
\mathbf{Q}_{31} & \mathbf{Q}_{32} & \mathbf{Q}_{33}
\end{array}\right]=\left[\begin{array}{lll}
\mathbf{I} & \mathbf{0} & \mathbf{0} \\
\mathbf{0} & \mathbf{I} & \mathbf{0} \\
\mathbf{0} & \mathbf{0} & \mathbf{I}
\end{array}\right] . \\
& \mathbf{Q}_{11}=\left[\begin{array}{lll}
\mathbf{T G}_{22} \mathbf{T}^{\prime}+\mathbf{D} & \mathbf{T G}_{22} & \mathbf{T Z B}
\end{array}\right]\left[\begin{array}{c}
\mathbf{D}^{-1} \\
-\mathbf{T}^{\prime} \mathbf{D}^{-1} \\
\mathbf{0}
\end{array}\right] \\
& =\left(\mathbf{T G}_{22} \mathbf{T}^{\prime}+\mathbf{D}\right) \mathbf{D}^{-1}-\mathbf{T G}_{22} \mathbf{T}^{\prime} \mathbf{D}^{-1}=\mathbf{I},
\end{aligned}
$$

For proving the diagonal elements $\mathbf{Q}_{i i}=\mathbf{I}$ and off-diagonal elements $\mathbf{Q}_{i i}=\mathbf{0}$, we repeatedly use the formula given in Equation [9]:

$$
\mathbf{G}_{22}=\mathbf{Z B Z}^{\prime}+k \mathbf{A}_{22} .
$$




$$
\begin{aligned}
\mathbf{Q}_{22} & =\left[\begin{array}{lll}
\mathbf{G}_{22} \mathbf{T}^{\prime} & \mathbf{G}_{22} & \mathbf{Z B}
\end{array}\right]\left[\begin{array}{c}
-\mathbf{D}^{-1} \mathbf{T} \\
\frac{1}{k} \mathbf{A}_{22}^{-1}+\mathbf{T}^{\prime} \mathbf{D}^{-1} \mathbf{T} \\
-\frac{1}{k} \mathbf{Z}^{\prime} \mathbf{A}_{22}^{-1}
\end{array}\right] \\
& =\mathbf{G}_{22} \mathbf{T}^{\prime}\left(-\mathbf{D}^{-1} \mathbf{T}\right)+\mathbf{G}_{22}\left(\frac{1}{k} \mathbf{A}_{22}^{-1}+\mathbf{T}^{\prime} \mathbf{D}^{-1} \mathbf{T}\right)-\frac{1}{k} \mathbf{Z B Z} \mathbf{Z}^{\prime} \mathbf{A}_{22}^{-1} \\
& =\mathbf{G}_{22}\left(-\mathbf{T}^{\prime} \mathbf{D}^{-1} \mathbf{T}+\frac{1}{k} \mathbf{A}_{22}^{-1}+\mathbf{T}^{\prime} \mathbf{D}^{-1} \mathbf{T}\right)-\frac{1}{k} \mathbf{Z B} \mathbf{Z}^{\prime} \mathbf{A}_{22}^{-1} \\
& =\frac{1}{k} \mathbf{G}_{22} \mathbf{A}_{22}^{-1}-\frac{1}{k} \mathbf{Z B} \mathbf{Z}^{\prime} \mathbf{A}_{22}^{-1}=\frac{1}{k}\left(\mathbf{G}_{22}-\mathbf{Z B} \mathbf{Z}^{\prime}\right) \mathbf{A}_{22}^{-1} \\
& =\frac{1}{k}\left(k \mathbf{A}_{22}\right) \mathbf{A}_{22}^{-1}=\mathbf{I},
\end{aligned}
$$

$$
\begin{aligned}
& =-\frac{1}{k} \mathbf{G}_{22} \mathbf{A}_{22}^{-1} \mathbf{Z}+\mathbf{Z}+\frac{1}{k} \mathbf{Z B Z} \mathbf{Z}^{\prime} \mathbf{A}_{22}^{-1} \mathbf{Z} \\
& =\mathbf{Z}-\frac{1}{k}\left(\mathbf{G}_{22}-\mathbf{Z B Z} \mathbf{Z}^{\prime}\right) \mathbf{A}_{22}^{-1} \mathbf{Z} \\
& =\mathbf{Z}-\frac{1}{k}\left(k \mathbf{A}_{22}\right) \mathbf{A}_{22}^{-1} \mathbf{Z}=\mathbf{Z}-\mathbf{Z}=\mathbf{0}
\end{aligned}
$$

$$
\begin{aligned}
& \mathbf{Q}_{32}=\left[\begin{array}{lll}
\mathbf{B} Z^{\prime} \mathbf{T}^{\prime} & \mathbf{B Z} & \mathbf{B}
\end{array}\right]\left[\begin{array}{c}
-\mathbf{D}^{-1} \mathbf{T} \\
\frac{1}{k} \mathbf{A}_{22}^{-1}+\mathbf{T}^{\prime} \mathbf{D}^{-1} \mathbf{T} \\
-\frac{1}{k} \mathbf{Z}^{\prime} \mathbf{A}_{22}^{-1}
\end{array}\right] \\
& =-\mathbf{B} \mathbf{Z}^{\prime} \mathbf{T}^{\prime} \mathbf{D}^{-1} \mathbf{T}+\mathbf{B} \mathbf{Z}^{\prime}\left(\frac{1}{k} \mathbf{A}_{22}^{-1}+\mathbf{T}^{\prime} \mathbf{D}^{-1} \mathbf{T}\right)-\frac{1}{k} \mathbf{B} \mathbf{Z}^{\prime} \mathbf{A}_{22}^{-1} \\
& =-\mathbf{B Z}^{\prime} \mathbf{T}^{\prime} \mathbf{D}^{-1} \mathbf{T}+\mathbf{B} \mathbf{Z}^{\prime} \mathbf{T}^{\prime} \mathbf{D}^{-1} \mathbf{T}+\frac{1}{k} \mathbf{B} \mathbf{Z}^{\prime} \mathbf{A}_{22}^{-1}-\frac{1}{k} \mathbf{B} \mathbf{Z}^{\prime} \mathbf{A}_{22}^{-1}=\mathbf{0},
\end{aligned}
$$

$$
\begin{aligned}
\mathbf{Q}_{33} & =\left[\begin{array}{lll}
\mathbf{B} \mathbf{Z}^{\prime} \mathbf{T}^{\prime} & \mathbf{B} \mathbf{Z}^{\prime} & \mathbf{B}
\end{array}\right]\left[\begin{array}{c}
\mathbf{0} \\
-\frac{1}{k} \mathbf{A}_{22}^{-1} \mathbf{Z} \\
\mathbf{B}^{-1}+\frac{1}{k} \mathbf{Z}^{\prime} \mathbf{A}_{22}^{-1} \mathbf{Z}
\end{array}\right] \\
& =-\frac{1}{k} \mathbf{B Z}^{\prime} \mathbf{A}_{22}^{-1} \mathbf{Z}+\mathbf{B}\left(\mathbf{B}^{-1}+\frac{1}{k} \mathbf{Z}^{\prime} \mathbf{A}_{22}^{-1} \mathbf{Z}\right) \\
& =\mathbf{I}-\frac{1}{k} \mathbf{B Z}^{\prime} \mathbf{A}_{22}^{-1} \mathbf{Z}+\frac{1}{k} \mathbf{B Z}^{\prime} \mathbf{A}_{22}^{-1} \mathbf{Z}=\mathbf{I} .
\end{aligned}
$$

To prove Equation [A3], we make use of Equation [A1], showing that the additive genetic effects of nongenotyped animals $\left(\mathbf{u}_{1}\right)$ is expressed in terms of the additive genetic effects of genotyped animals $\left(\mathbf{u}_{2}\right)$ and a deviation $(\mathbf{d})$. Because group 1 animals are not genotyped, our knowledge of the transmission of genes from group 2 to group 1 is based only on pedigree. Therefore, Equation [A1] would be the same if no animals were genotyped. The transmission matrix $\mathbf{T}$ is simply the regression coefficients based on the pedigree; that is, $\mathbf{T}=\mathbf{A}_{12} \mathbf{A}_{22}^{-1}$. Thus, the complete pedigree matrix $\mathbf{A}$ can be written as

$$
\operatorname{var}\left(\begin{array}{l}
\mathbf{u}_{1} \\
\mathbf{u}_{2}
\end{array}\right)=\mathbf{A} \sigma_{u}^{2}=\left[\begin{array}{cc}
\mathbf{T} \mathbf{A}_{22} \mathbf{T}^{\prime}+\mathbf{D} & \mathbf{T} \mathbf{A}_{22} \\
\mathbf{A}_{22} \mathbf{T}^{\prime} & \mathbf{A}_{22}
\end{array}\right] \sigma_{u}^{2} .
$$


The inverse of this matrix $\mathbf{A}$ is

$$
\mathbf{A}^{-1}=\left[\begin{array}{cc}
\mathbf{D}^{-1} & -\mathbf{D}^{-1} \mathbf{T} \\
-\mathbf{T}^{\prime} \mathbf{D}^{-1} & \mathbf{A}_{22}^{-1}+\mathbf{T}^{\prime} \mathbf{D}^{-1} \mathbf{T}
\end{array}\right],
$$

which can be verified by multiplying $\mathbf{A}$ by $\mathbf{A}^{-1}$ and getting $\mathbf{I}$, as shown in [A7] for matrix $\mathbf{G}$. Therefore, the submatrix of $\mathbf{A}^{-1}$ corresponding to group 1 animals (i.e., $\mathbf{A}^{11}$ ) is $\mathbf{D}^{-1}$, proving Equation $[\mathrm{A} 3] \mathbf{D}^{-1}=\mathbf{A}^{11}$.

The above formulas and equations were derived by M. E. Goddard and proved by Z. Liu.

\section{APPENDIX B: COMPARISON WITH A SINGLE-STEP GBLUP MODEL}

\section{A Single-Step SNP Model Without a Residual Polygenic Effect}

Under an assumption that fitted SNP markers can explain all genetic variation of a trait, Equation [3] is simplified to

$$
\mathbf{u}_{2}=\mathbf{Z g}
$$

A complete set of MME for the single-step model without an RPG effect becomes

$$
\left[\begin{array}{cccc}
\mathbf{X}^{\prime} \mathbf{X} & \mathbf{X}^{\prime} \mathbf{Z}_{p} & \mathbf{X}^{\prime} \mathbf{W}_{1} & \mathbf{X}^{\prime} \mathbf{W}_{2} \mathbf{Z} \\
\mathbf{Z}_{p}{ }^{\prime} \mathbf{X} & \mathbf{Z}_{p}{ }^{\prime} \mathbf{Z}_{p}+\mathbf{I} \delta & \mathbf{Z}_{p}{ }^{\prime} \mathbf{W}_{1} & \mathbf{Z}_{p}{ }^{\prime} \mathbf{W}_{2} \mathbf{Z} \\
\mathbf{W}_{1}{ }^{\prime} \mathbf{X} & \mathbf{W}_{1} \mathbf{Z}_{p} & \mathbf{W}_{1}{ }^{\prime} \mathbf{W}_{1}+\lambda \mathbf{A}^{11} & \lambda \mathbf{A}^{12} \mathbf{Z} \\
\mathbf{Z}^{\prime} \mathbf{W}_{2}{ }^{\prime} \mathbf{X} & \mathbf{Z}^{\prime} \mathbf{W}_{2}{ }^{\prime} \mathbf{Z}_{p} & \lambda \mathbf{Z}^{\prime} \mathbf{A}^{21} & \mathbf{Z}^{\prime} \mathbf{W}_{2}{ }^{\prime} \mathbf{W}_{2} \mathbf{Z}+\lambda \mathbf{Z}^{\prime}\left(\mathbf{A}^{22}-\mathbf{A}_{22}^{-1}\right) \mathbf{Z}+\lambda \mathbf{B}^{-1}
\end{array}\right]\left[\begin{array}{c}
\hat{\mathbf{b}} \\
\hat{\mathbf{p}} \\
\hat{\mathbf{u}}_{1} \\
\hat{\mathbf{g}}
\end{array}\right]=\left[\begin{array}{c}
\mathbf{X}^{\prime} \mathbf{y} \\
\mathbf{Z}_{p}{ }^{\prime} \mathbf{y} \\
\mathbf{W}_{1}{ }^{\prime} \mathbf{y} \\
\mathbf{Z}^{\prime} \mathbf{W}_{2}{ }^{\prime} \mathbf{y}
\end{array}\right] .
$$

Equation [B2] can be divided into 2 sets of equations that are solved iteratively:

$$
\begin{gathered}
{\left[\begin{array}{ccc}
\mathbf{X}^{\prime} \mathbf{X} & \mathbf{X}^{\prime} \mathbf{Z}_{p} & \mathbf{X}^{\prime} \mathbf{W}_{1} \\
\mathbf{Z}_{p}{ }^{\prime} \mathbf{X} & \mathbf{Z}_{p}{ }^{\prime} \mathbf{Z}_{p}+\mathbf{I} \delta & \mathbf{Z}_{p}{ }^{\prime} \mathbf{W}_{1} \\
\mathbf{W}_{1}{ }^{\prime} \mathbf{X} & \mathbf{W}_{1}{ }^{\prime} \mathbf{Z}_{p} & \mathbf{W}_{1}{ }^{\prime} \mathbf{W}_{1}+\lambda \mathbf{A}^{11}
\end{array}\right]\left[\begin{array}{c}
\hat{\mathbf{b}} \\
\hat{\mathbf{p}} \\
\hat{\mathbf{u}}
\end{array}\right]=\left[\begin{array}{c}
\mathbf{X}^{\prime} \mathbf{y} \\
\mathbf{Z}_{p}{ }^{\prime} \mathbf{y} \\
\mathbf{W}_{1}{ }^{\prime} \mathbf{y}-\lambda \mathbf{A}^{12} \mathbf{Z} \hat{\mathbf{g}}
\end{array}\right] \text { and }} \\
\\
\quad\left[\mathbf{Z}^{\prime} \mathbf{W}_{2}{ }^{\prime} \mathbf{W}_{2} \mathbf{Z}+\lambda \mathbf{Z}^{\prime}\left(\mathbf{A}^{22}-\mathbf{A}_{22}^{-1}\right) \mathbf{Z}+\lambda \mathbf{B}^{-1}\right] \hat{\mathbf{g}} \\
=\mathbf{Z}^{\prime} \mathbf{W}_{2}{ }^{\prime}\left(\mathbf{y}-\mathbf{X} \hat{\mathbf{b}}-\mathbf{Z}_{p} \hat{\mathbf{p}}\right)-\lambda \mathbf{Z}^{\prime} \mathbf{A}^{21} \hat{\mathbf{u}}_{1} .
\end{gathered}
$$

It can be proven that MME of our single-step SNP model without an RPG are identical to those by Legarra and Ducrocq (2012), when the nongenetic random effect $\mathbf{p}$ of our single-step SNP model [Eq. 1] is ignored in the comparison of MME of both models.

\section{Comparison with the Single-Step GBLUP Model by Legarra and Ducrocq (2012)}

Because the terms for conventional parts in MME are identical between their SSGblup model (Legarra and Ducrocq, 2012) and our single-step SNP model with a RPG effect [Eq. 1], we compare only the genomic terms in both models. In Legarra and Ducrocq's model a genomic contribution to RHS of MME is defined as

$$
\alpha_{u} \hat{\phi}-\alpha_{u} \hat{\gamma}=\alpha_{u} \mathbf{A}_{22}^{-1} \hat{\mathbf{u}}_{2}-\alpha_{u} \mathbf{G}^{-1} \hat{\mathbf{u}}_{2},
$$


and SNP effects are estimated (Strandén and Garrick, 2009):

$$
\hat{\mathrm{g}}=\mathbf{D Z}^{\prime} \mathbf{G}^{-1} \hat{\mathbf{u}}_{2} .
$$

Equations [B5] and [B6] can be rewritten using the terms of our single-step SNP model:

$$
\begin{aligned}
\lambda \hat{\phi}-\lambda \hat{\gamma} & =\lambda \mathbf{A}_{22}^{-1} \hat{\mathbf{u}}_{2}-\lambda \mathbf{G}_{22}^{-1} \hat{\mathbf{u}}_{2}, \\
\hat{\mathbf{g}} & =\mathbf{B Z}^{\prime} \mathbf{G}_{22}^{-1} \hat{\mathbf{u}}_{2} .
\end{aligned}
$$

The corresponding terms of our single-step SNP model [Eq. 1] are Equations [B9] and [B10], respectively:

$$
\begin{gathered}
\lambda \mathbf{A}_{22}^{-1} \hat{\mathbf{u}}_{2}-\frac{1}{k} \lambda \mathbf{A}_{22}^{-1} \hat{\mathbf{a}}_{2} \text { and } \\
\hat{\mathbf{g}}=\frac{1}{k} \mathbf{B} \mathbf{Z}^{\prime} \mathbf{A}_{22}^{-1} \hat{\mathbf{a}}_{2} .
\end{gathered}
$$

To prove that Equations [B9] and [B10] of our singlestep SNP model are equal to Equations [B7] and [B8], respectively, of the single-step GBLUP model by Legarra and Ducrocq (2012), we need the following (co) variances:

$$
\operatorname{var}\left(\mathbf{a}_{2}\right)=k \mathbf{A}_{22} \sigma_{u}^{2},
$$

$$
\operatorname{var}\left(\mathbf{u}_{2}\right)=\mathbf{G}_{22} \sigma_{u}^{2},
$$

$$
\operatorname{cov}\left(\mathbf{a}_{2}, \mathbf{u}_{2}\right)=\operatorname{cov}\left(\mathbf{a}_{2}, \mathbf{Z g}+\mathbf{a}_{2}\right)=\operatorname{var}\left(\mathbf{a}_{2}\right)=k \mathbf{A}_{22} \sigma_{u}^{2} .
$$

The RPG $\mathbf{a}_{2}$ can be estimated from $\hat{\mathbf{u}}_{2}$ with

$$
\hat{\mathbf{a}}_{2}=\operatorname{cov}\left(\mathbf{a}_{2}, \mathbf{u}_{2}\right)\left[\operatorname{var}\left(\mathbf{u}_{2}\right)\right]^{-1} \hat{\mathbf{u}}_{2}=k \mathbf{A}_{22} \mathbf{G}_{22}^{-1} \hat{\mathbf{u}}_{2} .[\mathrm{B} 14]
$$

Multiplying both sides of Equation [B14] with $\frac{1}{k} \mathbf{A}_{22}^{-1}$ gives

$$
\frac{1}{k} \mathbf{A}_{22}^{-1} \hat{\mathbf{a}}_{2}=\mathbf{G}_{22}^{-1} \hat{\mathbf{u}}_{2} .
$$

From Equation [B15], we can see that the 2 single-step models have equivalent formulas for calculating the genomic contributions [B7] and [B9] and for estimating SNP effects [B8] and [B10].

Although in theory the 2 models are equivalent, the computing algorithms are different. It can be clearly seen that the formulas for the genomic contribution to RHS of MME differ between the 2 models, [B9] versus [B7]. For SNP effect estimation, our single-step SNP model [1] uses $\frac{1}{k} \mathbf{A}_{22}^{-1} \hat{\mathbf{a}}_{2}$ instead of $\mathbf{G}_{22}^{-1} \hat{\mathbf{u}}_{2}$, which can be seen in comparing [B10] and [B8]. 